\title{
Synthesis of 4-O-Alkylated $\mathrm{N}$-Acetylneuraminic Acid Derivatives
}

\author{
Emil Johansson, Rémi Caraballo, and Mikael Elofsson*
}

Cite This: J. Org. Chem. 2021, 86, 9145-9154

Read Online

ABSTRACT: The synthesis of 4-O-alkyl analogues of $\mathrm{N}$-acetylneuraminic acid (Neu5Ac) and the scope of the reaction are described. Activated alkyl halides and sulfonates and primary alkyl iodides give products in useful yields. The utility of the methodology is exemplified using a thiophenyl Neu5Ac building block to synthesize a 4-O-alkyl DANA analogue. These results expand the toolbox of Neu5Ac chemistry with value in drug discovery and for the design of novel tools to study the biology of Neu5Ac lectins.

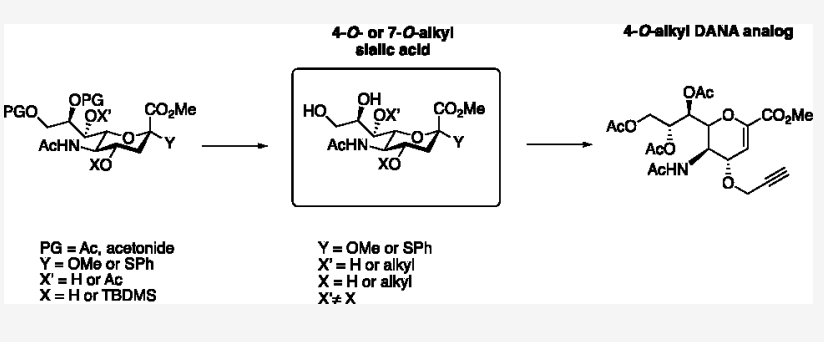

$N$-Acetylneuraminic acid (Neu5Ac, 1, Figure 1) is typically found at the terminal end of glycolipids and glycoproteins that
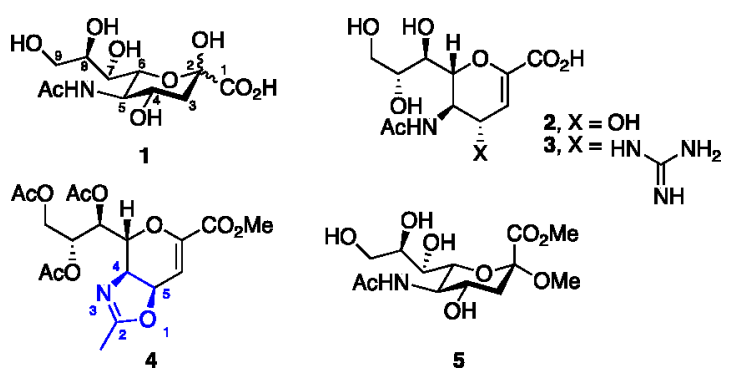

Figure 1. Structure of NeuSAc and DANA analogues.

decorate the surfaces of all mammalian cell types. Neu5Ac is involved in mediating or modulating a variety of physiological and pathophysiological processes. ${ }^{1}$ One of the most wellknown roles of NeuSAc is in the replication cycle of the influenza virus. ${ }^{2}$ Accordingly, substantial efforts have been placed on the development of Neu5Ac-based antivirals, ${ }^{3}$ where modifications of the C4-position of 2-deoxy-2,3-didehydro- $N$ acetylneuraminic acid (DANA, 2, Figure 1) have been of central importance. $^{4-12}$ This culminated in the development of Relenza (3, Figure 1), a C4-modified analogue of $\mathbf{2}$ designed to mimic the transition state of $\mathbf{1}$ during the neuraminidase catalyzed hydrolysis reaction required for release of virus progeny from infected cells. ${ }^{5}$ C4-modified analogues of $\mathbf{2}$ including nitrogen, ${ }^{4}$ sulfur, ${ }^{4}$ and deoxygenated ${ }^{7}$ compounds are efficiently accessed via selective ring opening at position 5 of the allylic oxazoline of 2,3-didehydro- $N$-acetylneuraminic acid (4, Figure 1). ${ }^{4}$ However, in the case of oxygen nucleophiles, opening occurs at position 2 of the oxazoline ring. ${ }^{7}$ Hence, the method is not applicable to the synthesis of 4-O-modified analogues of $\mathbf{2}$ (or $\mathbf{1}$ ) with retained stereochemistry. C4-deoxy and C4-nitrogen analogues of 1 can, however, be accessed using the ring-opening methodology but require reinstallment of the glycosidic bond, which produces two stereoisomers of equal proportions. ${ }^{13,14}$

The interest in Neu5Ac analogues and their roles in biological systems is constantly increasing. Therefore, efficient methods that allow site-selective modifications of the Neu5Actemplate are of great utility for studying Neu5Ac biology and for drug discovery. Methods to selectively access C4-modified analogues of $\mathbf{1}$ are scarce, with relatively few reported examples. These include carba, ${ }^{15,16}$ keto, ${ }^{16}$ ether, ${ }^{14,17-20}$ nitrogen, ${ }^{13}$ and deoxygenated ${ }^{21}$ derivatives. A potential drawback in the development of direct methods could be the competing formation of intramolecular lactams $\mathrm{s}^{22-24}$ and lactones ${ }^{25}$ that occur under both basic and acidic conditions. To date, examples of selectively 4-O-modified Neu5Ac analogues include 4-O-Ac, -benzyl, ${ }^{26-30}$-allyl, ${ }^{23}$-silyl, ${ }^{15}$ -methyl, ${ }^{14,17,18}$-ethyl, ${ }^{18}$-cyanomethyl, ${ }^{19}$ and -tert-butoxyace$\operatorname{tate}^{20}$ groups. The electrophiles used to produce these 4-Omodified analogues all have in common that they are activated, highly reactive, and (with a few exceptions) lack the presence of $\beta$-hydrogens. Further, the commercial availability of suitable electrophiles remains limited. Herein, we set out to study the scope and the 4-O-alkylation of Neu5Ac.

In an ongoing research project, we were interested in studying the potential of 4-O-alkyl analogues of 5 (Figure 1 ) as probes targeting cell attachment during adenovirus ${ }^{31-33}$ and coxsackievirus infections. ${ }^{34,35}$ We hypothesized that 6 (Scheme 1) would be a suitable substrate to study $O$-alkylation. This previously described protective group strategy is straightforward, high yielding, and allows removal of the protective groups in a final single step. ${ }^{15}$ Propargyl bromide was selected

Received: January 30, 2021

Published: June 17, 2021 
Scheme 1. Synthesis of Neu5Ac Derivatives 13, 15, and 18 Selectively Protected at the 7-, 8-, and 9-Positions
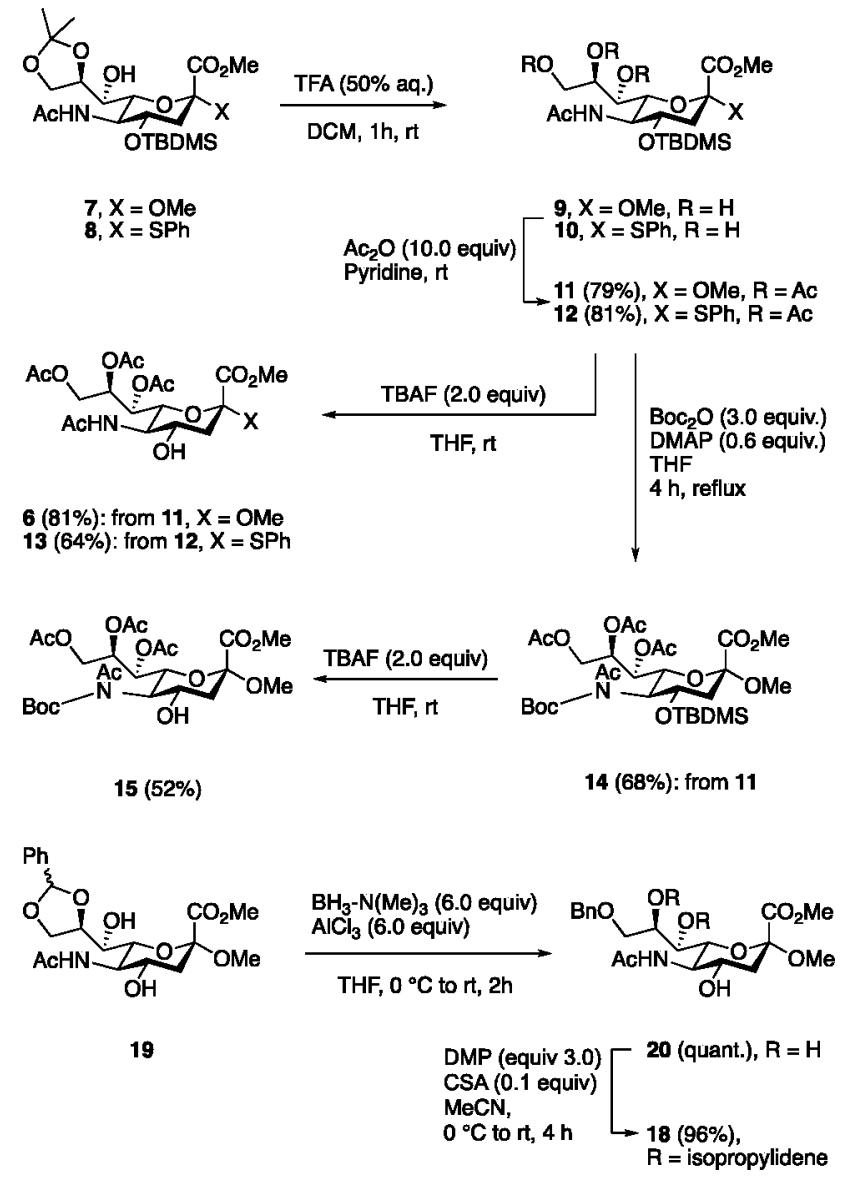

as the model electrophile. The lack of $\beta$-protons minimizes the competing E2 reaction, thus providing a fair measure of the effectiveness of the $S_{N} 2$ reaction. In addition, the generated alkynyl product can be further modified under mild conditions. ${ }^{36,37}$ Compound $\mathbf{5}^{38}$ (Figure 1) was obtained from 1 and then converted by standard methods to the known derivative $7^{39}$ (Scheme 1). Treatment of 7 with wet trifluoroacetic acid in DCM afforded 9, which upon acetylation gave the fully protected derivative 11 in $79 \%$ yield over two steps. Treatment of $\mathbf{1 1}$ with TBAF in THF afforded $\mathbf{6}$ in $81 \%$ yield.

Attempts to alkylate 6 using $\mathrm{Ba}(\mathrm{OH})_{2} / \mathrm{BaO}$ in $\mathrm{DMF},{ }^{23,30,40}$ $\mathrm{K}_{2} \mathrm{CO}_{3}$ in $\mathrm{THF}$, or $\mathrm{CsCO}_{3}$ in $\mathrm{MeCN}$, resulted in minimal amounts of $\mathbf{1 6}$. However, promising observations were made in THF using KHMDS or $\mathrm{NaH}$, with $\mathrm{NaH}$ providing superior conversion and product formation. Standard $O$-alkylation conditions were screened by treating 6 with $\mathrm{NaH}$ on ice prior to the addition of propargyl bromide (entries 1 and 2, Table 1). In DMF, this resulted in nearly complete decomposition and only trace amounts of 16 (entry 1). However, in THF, the 4-O-propargylated derivative 17 was isolated in $50 \%$ yield over two steps (entry 2 ). The $O$ deacetylation was performed to compensate for the formation of hydrolyzed species during the reaction (Figure S1) and, thus, facilitate isolation of the desired $4-O$-alkyl product. The $O$-deacetylation of purified $\mathbf{1 6}$ gave $\mathbf{1 7}$ in $85 \%$ yield, the value that was used to estimate the yield for the $O$-alkylation (Table 1).
Table 1. Screening and Optimization of Reaction Conditions

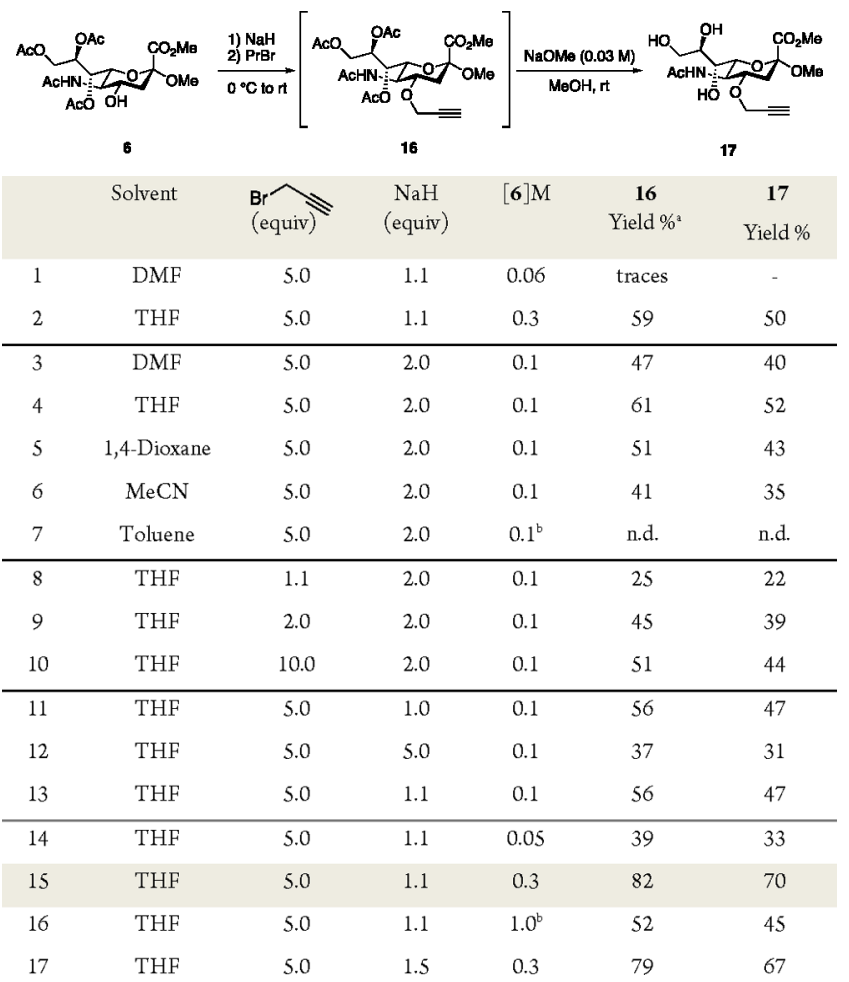

${ }^{a}$ Estimated yields are based on the isolated yield $(85 \%)$ of the $O$ deacetylated $17 .{ }^{b}$ Solubility issues; n.d. = not determined.

Alkoxide formation was studied by mixing compound $\mathbf{6}$ with $\mathrm{NaH}$ in DMF- $d_{7}$ and in THF- $d_{8}$, respectively, and recording ${ }^{1} \mathrm{H}$ NMR spectra at two different $10 \mathrm{~min}$ and $1 \mathrm{~h}$ (Figure S2AF). Within $10 \mathrm{~min}$, compound 6 was essentially consumed in DMF- $d_{7}$, resulting in a complex mixture of products (Figure $\mathrm{S} 2 \mathrm{~A}, \mathrm{~B})$. In contrast, only minimal signs of degradation were observed in THF- $d_{8} 10$ min postaddition of $\mathrm{NaH}$ (Figure S2D,E), and the majority of $\mathbf{6}$ was largely intact after $1 \mathrm{~h}$ (Figure S2F).

This prompted us to reverse the addition order, and compound 6 was mixed with propargyl bromide in the selected solvent on ice before adding $\mathrm{NaH}$. Furthermore, the stoichiometry of $\mathrm{NaH}$ was increased from 1.1 to 2.0 equiv to ensure complete deprotonation of both the hydroxyl and acetamide of 6 . These modifications drastically improved the yield of $\mathbf{1 7}$ in DMF (40\%; entry 3, Table 1), while no significant effect was observed in THF ( $52 \%$ yield; entry 4 ). This highlights the importance of avoiding preformation of the alkoxide in DMF. The 4-O-alkylated product 17 was confirmed by $2 \mathrm{D}$ NMR analysis and by treatment with acetic anhydride in pyridine, which afforded 16 in $60 \%$ yield.

Common solvents for $\mathrm{O}$-alkylation reactions were screened, and the yields of 17 were lower in both 1,4-dioxane (43\%; entry 5, Table 1) and $\mathrm{MeCN}$ (35\%; entry 6) compared to the reference reaction (entry 4$)$. The reaction in toluene (entry 7 ) was slow, with incomplete conversion after $72 \mathrm{~h}$ of stirring, likely due to poor solubility, and was not processed further. Decreasing the stoichiometry of propargyl bromide to 1.1 and 2.0 equiv afforded $\mathbf{1 7}$ in $22 \%$ and $39 \%$ yields, respectively (entries 8 and 9), while increased stoichiometry gave 17 in 
$44 \%$ yield (10 equiv; entry 10) and resulted in a larger concentration of side products.

Reduced stoichiometry of $\mathrm{NaH}$ gave $\mathbf{1 7}$ in $47 \%$ yield (1.0 equiv; entry 11, Table 1) with incomplete conversion, while increased stoichiometry provided $\mathbf{1 7}$ in $31 \%$ yield (5.0 equiv; entry 12) with larger amounts of side products, suggesting the stoichiometry of $\mathrm{NaH}$ should be greater than one but less than two equivalents to ensure complete conversion and minimize the formation of side products. Indeed, 1.1 equiv of $\mathrm{NaH}$ gave a clean reaction and complete conversion, albeit without improvement of the yield (47\%; entry 13). Decreased substrate concentration gave $\mathbf{1 7}$ in $33 \%$ yield $(0.05 \mathrm{M}$; entry 14$)$. Pleasingly, increased concentration produced 17 in $70 \%$ yield (0.3 M; entry 15), corresponding to a $35 \%$ improvement compared to the reference reaction. Higher concentration was associated with solubility issues but provided 17 in $45 \%$ yield (1.0 M; entry 16). With the optimized conditions in hand, the stoichiometry of $\mathrm{NaH}$ was adjusted to 1.5 equiv as the conversion was incomplete in some reactions when using 1.1 equiv. This resulted in complete conversion of 6 , providing 17 in $67 \%$ yield (entry 17 ). To conclude, the optimal conditions are a concentration of $0.3 \mathrm{M}$ (in THF), 5.0 equiv of propargyl bromide, and $1.1-1.5$ equiv of $\mathrm{NaH}$.

In an attempt to further improve the yields of the $O$ alkylation, compounds $\mathbf{1 5}$ and $\mathbf{1 8}$ were prepared (Scheme 1). Compound 15 with its tertiary amide renders it resistant toward potential side products arising from lactamization. ${ }^{22-24}$ Compound $\mathbf{1 5}$ was accessed from $\mathbf{1 1}$ by treatment with Boc anhydride and DMAP in THF followed by cleavage of the TBDMS group using TBAF (Scheme 1). Surprisingly, the reactivity of $\mathbf{1 5}$ was completely abolished toward $O$-alkylation (entry 1, Table 2).

Prolonged reaction times $(2.5 \mathrm{~h})$, heating $\left(60^{\circ} \mathrm{C}\right.$ for $16 \mathrm{~h}$, with a heating mantle), and irradiation in a microwave reactor $\left(100{ }^{\circ} \mathrm{C}\right.$ for $20 \mathrm{~min}$ ) were inefficient in causing conversion. Compound 18 was prepared from the known derivative $19^{41}$ including selective reduction with borane-trimethyl amine and aluminum chloride in THF affording $\mathbf{2 0}$ in quantitative yield that upon treatment with 2,2-dimethoxypropane and camphor sulfonic acid in $\mathrm{MeCN}$ gave the 9-O-benzyl-7,8-acetonide protected 18 in $96 \%$ yield. This protective group strategy is orthogonal allowing site-selective removal and functionalization of the glycerol side chain (C7, C8, and C9). Further, the protective groups have increased resistance toward hydrolysis under basic conditions. Upon $O$-alkylation 18 gave 22 in 57\% yield (entry 2). Compound 13 was prepared in analogueous manner to $\mathbf{6}$ (Scheme 1), and upon $O$-alkylation afforded 23 in 74\% yield (entry). Compound 23, and analogues thereof, significantly broaden the scope due to their potential for modifications at the $\mathrm{C} 2$-position via glycosylation, or elimination to access 4-O-alkyl DANA analogues. ${ }^{19}$ The developed conditions were applied to synthetic intermediates 7 and $\mathbf{8}$ which provided $\mathbf{2 4}$ and $\mathbf{2 5}$ in $57 \%$ and $71 \%$ yields, respectively (entries 4 and 5). Thus, supporting access to 7-Oalkylated species. Synthetic intermediates 26 and 27 selectively afforded the 4-O-alkylated products 28 and 29 in 31\% and $49 \%$ yields (entries 6 and 7), respectively. Thus, significantly decreasing the number of steps to access 4-O-alkylated analogues of 18 .

Representative examples of commercial alkyl halides and sulfonates were then screened to study the scope of the 4-Oalkylation of 6 (Figure 2). As expected, the activated alkyl bromides benzyl bromide, allyl bromide, and ethyl bromoace-
Table 2. $O$-alkylation of Diversely Protected Neu5Ac Building Blocks ${ }^{a}$

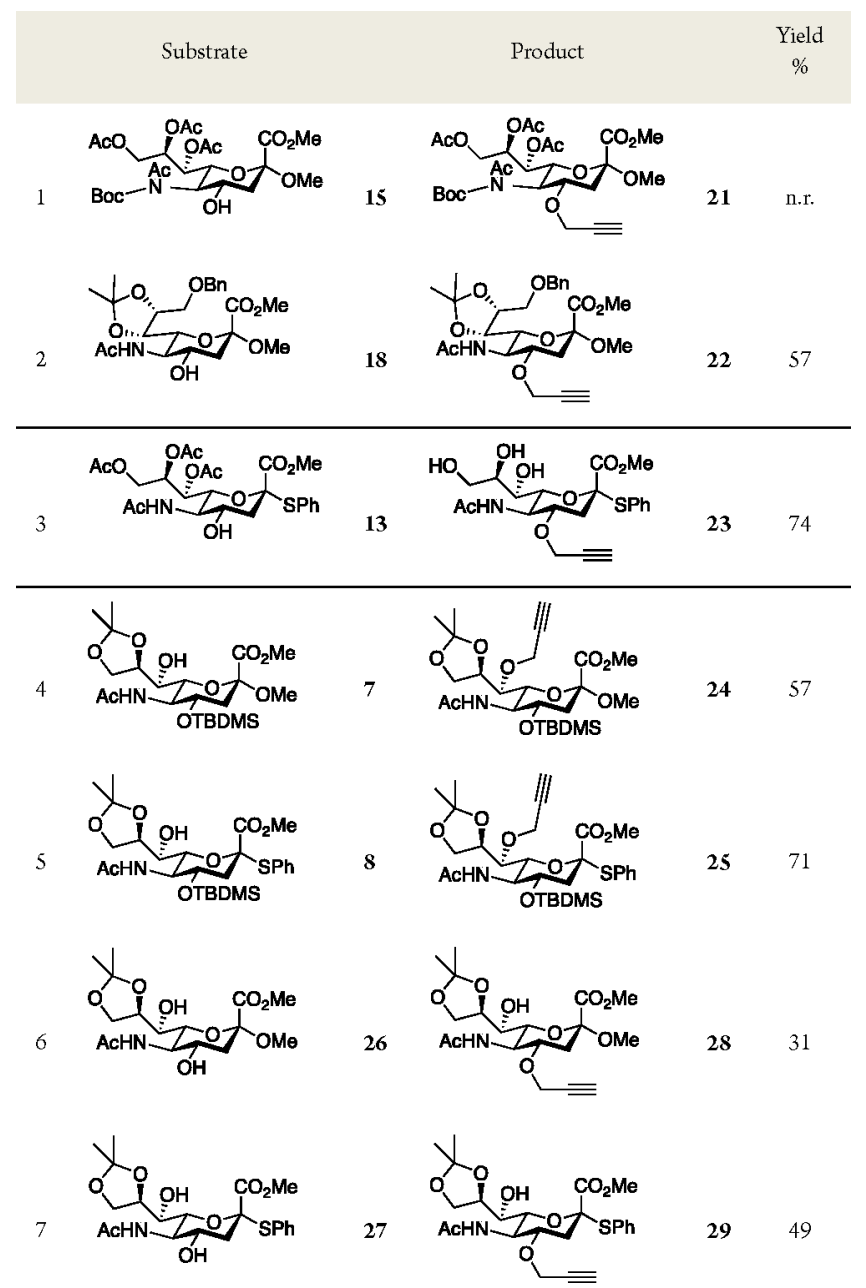

${ }^{a}$ All reactions were conducted in THF $(0.3 \mathrm{M}$ substrate $)$ and performed by treating a stirred solution of 5.0 equiv of propargyl bromide and substrate with 1.1-1.5 equiv of $\mathrm{NaH}$ (specific details in Supporting Information). n.r. $=$ no reaction. Yield over two steps.

tate afforded the corresponding 4-O-ethers 30 (62\% yield), 31 ( $52 \%$ yield), and 32 (78\% yield) (Figure 2$)$, respectively. An initial reaction with 6-iodo-1-hexyne gave 33 in a mere $10 \%$ yield. Dipolar aprotic solvents are known to increase the rate of substitution due to their ability to solvate cations, ${ }^{42}$ and the use of DMF indeed afforded 33 in $45 \%$ yield. Using 6-chloro-1hexyne resulted in trace amounts of 33 , and the addition of TBAI, or KI, did not result in the isolation of $\mathbf{3 3}$ in either THF or DMF. Upon $\mathrm{O}$-alkylation 5-bromo-1-pentene afforded 34 in $17 \%$ yield. Ethyl tosylate gave 35 in poor yield (8\%) with substantial amounts of hydrolyzed starting material. However, propargyl mesylate gave $\mathbf{1 7}$ in $58 \%$ yield, supporting the use of activated alkyl sulfonates. Last, we attempted to substitute 2bromopropane, which resulted in trace amounts of 36 (Figure 2 ), in line with the fact that $2^{\circ}$ halides are less reactive in $O$ alkylation reactions due to excess $\beta$-protons favoring an E2 pathway over the desired substitution reaction. ${ }^{43}$

To exemplify the utility of the developed methodology, we purified intermediate 37 and treated it with TfOH and NIS in $\mathrm{DCM}^{44}$ affording the 4-O-propargyl DANA analogue 38 in $87 \%$ yield (Scheme 2), thus confirming access to 4 -O-alkyl 


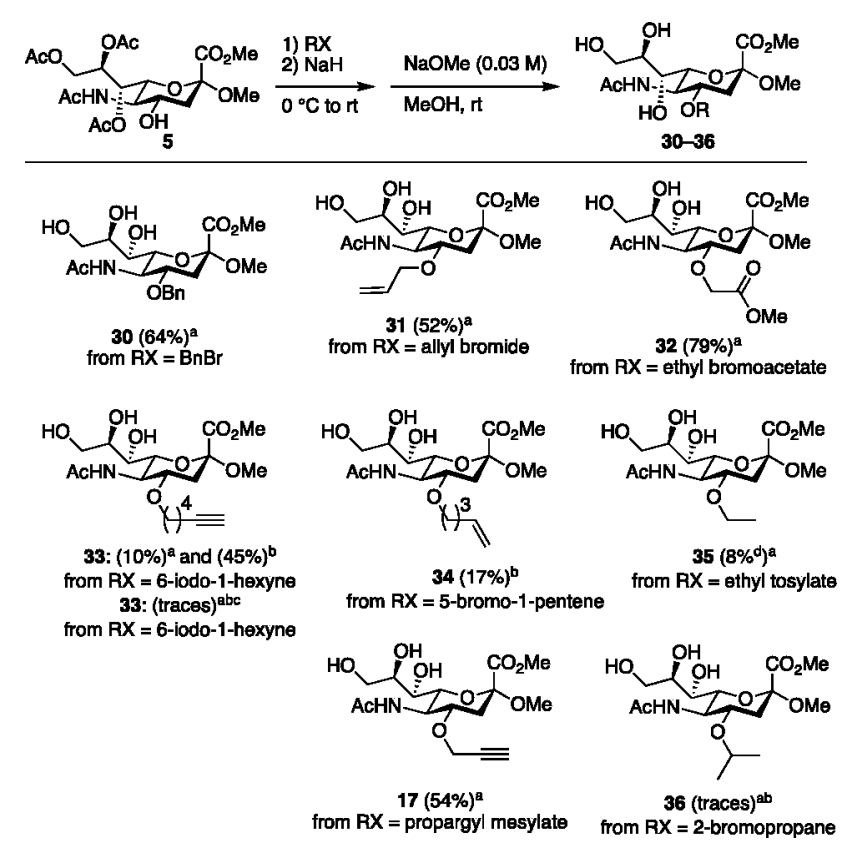

Figure 2. Scope of 4-O-alkylation. Outline of reaction (top). Synthesized substrates and used electrophiles (=RX). Footnote a represents THF as a solvent. Footnote b represents DMF as a solvent. Footnote $\mathrm{c}$ represents $\mathrm{KI}$ or TBAI as additives. Footnote $\mathrm{d}$ represents $80 \%$ pure.

DANA analogues, ${ }^{19}$ via C3-elimination. These compounds have potential as antivirals toward human parainfluenza type$1.45-50$

Scheme 2. Synthesis of a 4-O-Propargyl DANA Analogue

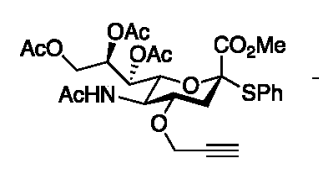

37
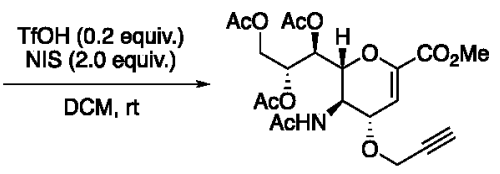

$38(87 \%)$
In summary, we have systematically studied $O$-alkylation of Neu5Ac derivatives and provided insights into the scope of the reaction for preparation of tool compounds and starting points for drug discovery. ${ }^{51}$

\section{EXPERIMENTAL SECTION}

General Chemical Procedures. ${ }^{1} \mathrm{H}$ NMR and ${ }^{13} \mathrm{C}$ NMR spectra were recorded with a Bruker DRX-400 spectrometer at 400 and 100 $\mathrm{MHz}$, respectively, or with a Bruker DRX-600 spectrometer at 600 and $150 \mathrm{MHz}$, respectively. NMR experiments were conducted at 298 $\mathrm{K}$ in $\mathrm{CD}_{3} \mathrm{OD}$ (residual solvent peak $=3.31 \mathrm{ppm}, \delta \mathrm{H}$ and $49.00 \mathrm{ppm}$, $\delta \mathrm{C}$ ) or $\mathrm{CDCl}_{3}$ (residual solvent peak $=7.26 \mathrm{ppm}, \delta \mathrm{H}$ and $77.16 \mathrm{ppm}$, $\delta \mathrm{C})$. Liquid chromatography-mass spectrometry (LC-MS) data were recorded by detecting positive/negative ions (electrospray ionization, ESI) on an Agilent 1290 Infinity II-6130 Quadrupole using $\mathrm{H}_{2} \mathrm{O} / \mathrm{CH}_{3} \mathrm{CN}$ ( $0.1 \%$ formic acid) as the eluent system or on an Agilent 1290 Infinity-6150 Quadrupole using YMC Triart C18 (1.9 $\mu \mathrm{m}, 20 \mathrm{~mm} \times 50 \mathrm{~mm}$ column) and $\mathrm{H}_{2} \mathrm{O} / \mathrm{CH}_{3} \mathrm{CN}$ (0.1\% formic acid) as the eluent system. High-resolution mass spectrometry (HRMS) data were recorded on an Agilent 1290 binary LC System connected to an Agilent 6230 Accurate-Mass Time-of-Flight (TOF) LC-MS (ESI+), which was calibrated with Agilent G1969-85001 ES-TOF Reference Mix containing ammonium trifluoroacetate, purine, and hexakis $(1 H, 1 H, 3 H$-tetrafluoropropoxy)phosphazine in 90:10 $\mathrm{CH}_{3} \mathrm{CN} / \mathrm{H}_{2} \mathrm{O}$. Semipreparative high-performance liquid chromatography (HPLC) was performed on a Gilson system using a YMC-Actus Triart C18, $12 \mathrm{~nm}, \mathrm{~S}-5 \mu \mathrm{m}, 250 \mathrm{~mm} \times 20.0 \mathrm{~mm}$ with a flow rate of 20 $\mathrm{mL} \min ^{-1}$, detection at $214 \mathrm{~nm}$, and eluent system $A$ with aqueous $0.005 \%$ formic acid, and B with $\mathrm{CH}_{3} \mathrm{CN} 0.005 \%$ formic acid. Thinlayer chromatography (TLC) was performed on silica gel 60 F254 (Merck) with detection under ultraviolet (UV) light and/or development with $5 \% \mathrm{H}_{2} \mathrm{SO}_{4}$ in $\mathrm{EtOH}$ and heat. Flash chromatography was performed using a Biotage Isolera One system and purchased prepacked silica gel cartridges (Biotage Sfär Silica). Lyophilization was performed by freezing the diluted $\mathrm{CH}_{3} \mathrm{CN}$ /water solutions in a dry ice-acetone bath or liquid nitrogen and then employing an Alpha 3-4 LSCbasic freeze-dryer. Organic solvents were dried using a Glass Contour Solvent System (SG Water USA). All commercial reagents were used as received. All target compounds were $\geq 95 \%$ pure according to HPLC UV traces, unless otherwise noted.

General Procedure for O-Alkylation (GP1): Exemplified with the Synthesis of 16. An oven-dried vial was charged with a magnetic stirring bar and compound $6(40 \mathrm{mg}, 0.086 \mathrm{mmol})$. The vial was placed under nitrogen, and THF $(0.3 \mathrm{~mL})$ followed by propargyl bromide ( $48 \mu \mathrm{L}, 0.43 \mathrm{mmol}, 5$ equiv) were added. The mixture was cooled to an ice-bath temperature, and $\mathrm{NaH}(3.8 \mathrm{mg}, 0.095 \mathrm{mmol}, 1.1$ equiv) was added in portions. After $10 \mathrm{~min}$, the reaction was allowed to perform at room temperature for an additional $2 \mathrm{~h}$ (monitored by TLC/EtOAc; $R_{f}=0.43$ ). After completion, the reaction was quenched by the addition of a few drops of sat. aq $\mathrm{NH}_{4} \mathrm{Cl}$, and the solvents were removed under reduced pressure. The crude product was directly used in the deacetylation step, unless otherwise noted.

General Procedure for Deacetylation (GP2): Exemplified with the Synthesis of 17 . Crude 16 was dissolved in dry methanol ( $4 \mathrm{~mL})$, and sodium, methoxide $(28 \mathrm{mg}, 0.52 \mathrm{mmol}, 6$ equiv) was added in portions. The reaction was allowed to perform at room temperature for $1 \mathrm{~h}$ (monitored by TLC: Tol/ $\mathrm{CH}_{3} \mathrm{OH}(4: 1, \mathrm{v} / \mathrm{v}) ; R_{f}$ $=0.40$ ). The mixture was then neutralized with Amberlyst $\mathrm{H}^{+}$-form, filtered, and concentrated to dryness. The compound was purified using flash chromatography $\left(\mathrm{Tol} / \mathrm{CH}_{3} \mathrm{OH}\right.$, gradient 5-20\%) to give compound 17.

Chemical Synthesis. Methyl (Methyl 5-acetamido-4-O-(tertbutyldimethylsilyl)-3,5-dideoxy-8,9-0-isopropylidene-D-glycero- $\alpha$ D-galacto-2-nonulopyranosid)onate (7). Compound 7 (1.39 g, $2.84 \mathrm{mmol}$, white foam) was synthesized in $71 \%$ yield following the procedure described in ref $1 .{ }^{1} \mathrm{H}$ NMR $\left(\mathrm{CDCl}_{3}, 600 \mathrm{MHz}\right): \delta 5.19$ (d, $1 \mathrm{H}, J=7.3 \mathrm{~Hz}), 4.32(\mathrm{q}, 1 \mathrm{H}, J=6.4 \mathrm{~Hz}), 4.25(\mathrm{~d}, 1 \mathrm{H}, J=5.0 \mathrm{~Hz})$, $4.12(\mathrm{dd}, 1 \mathrm{H}, J=8.5,6.3 \mathrm{~Hz}), 4.06(\mathrm{dd}, 1 \mathrm{H}, J=8.4,6.3 \mathrm{~Hz}), 3.85-$ $3.73(\mathrm{~m}, 2 \mathrm{H}), 3.80(\mathrm{~s}, 3 \mathrm{H}), 3.57(\mathrm{t}, 1 \mathrm{H}, J=5.7 \mathrm{~Hz}), 3.40(\mathrm{~s}, 3 \mathrm{H})$, $3.39(\mathrm{~d}, 1 \mathrm{H}, J=10.1 \mathrm{~Hz}), 2.59(\mathrm{dd}, 1 \mathrm{H}, J=12.8,4.2 \mathrm{~Hz}), 2.02(\mathrm{~s}$, $3 \mathrm{H}), 1.82(\mathrm{dd}, 1 \mathrm{H}, J=12.5,10.9 \mathrm{~Hz}), 1.37(\mathrm{~s}, 3 \mathrm{H}), 1.36(\mathrm{~s}, 3 \mathrm{H}), 0.87$ (s, 9H), $0.10(\mathrm{~s}, 3 \mathrm{H}), 0.09(\mathrm{~s}, 3 \mathrm{H}) .{ }^{13} \mathrm{C}\left\{{ }^{1} \mathrm{H}\right\}$ NMR $\left(\mathrm{CDCl}_{3}, 150\right.$ $\mathrm{MHz}): \delta 172.5,169.1,108.7,99.3,75.4,74.4,70.1,69.0,67.0,53.7$, 52.5, 51.8, 40.6, 27.0, 25.7, 23.3, 18.0, -3.9, -4.6. HRMS (ESI-TOF) $m / z:[\mathrm{M}+\mathrm{Na}]^{+}$calcd for $\mathrm{C}_{22} \mathrm{H}_{41} \mathrm{NO}_{9} \mathrm{SiNa}, 514.2443$; found, 514.2467.

Methyl (Phenyl 5-acetamido-4-O-(tert-butyldimethylsilyl)-3,5dideoxy-8,9-O-isopropylidene-2-thio- $D$-glycero- $\alpha$-D-galacto-2nonulopyranosid)onate ( 8 ). ${ }^{2}$ Compound 8 (0.78 g, $1.89 \mathrm{mmol}$, paleyellow foam) was synthesized in $73 \%$ yield following the procedure described in ref $2 .{ }^{1} \mathrm{H}$ NMR $\left(\mathrm{CD}_{3} \mathrm{OD}, 600 \mathrm{MHz}\right): \delta 7.58(\mathrm{dd}, 2 \mathrm{H}, J=$ 8.3, $1.4 \mathrm{~Hz}, 2 \mathrm{H}), 7.42(\mathrm{t}, 1 \mathrm{H}, J=7.2 \mathrm{~Hz}), 7.36(\mathrm{t}, 2 \mathrm{H}, J=7.6 \mathrm{~Hz})$, $4.15(\mathrm{q}, 1 \mathrm{H}, J=6.7 \mathrm{~Hz}), 3.98(\mathrm{dd}, 1 \mathrm{H}, J=8.2,6.3 \mathrm{~Hz}), 3.88(\mathrm{dd}, 1 \mathrm{H}$, $J=8.5,6.7 \mathrm{~Hz}), 3.91-3.82(\mathrm{~m}, 1 \mathrm{H}), 3.77-3.68(\mathrm{~m}, 1 \mathrm{H}), 3.50(\mathrm{~d}, 1 \mathrm{H}$, $J=7.1 \mathrm{~Hz}), 3.48(\mathrm{~s}, 3 \mathrm{H}), 3.45(\mathrm{~d}, 1 \mathrm{H}, J=10.6 \mathrm{~Hz}), 2.72(\mathrm{dd}, 1 \mathrm{H}, J=$ 12.7, $4.7 \mathrm{~Hz}), 1.92(\mathrm{~s}, 3 \mathrm{H}), 1.76(\mathrm{dd}, 1 \mathrm{H}, J=12.7,11.0 \mathrm{~Hz}), 1.31(\mathrm{~s}$, $3 \mathrm{H}), 1.23(\mathrm{~s}, 3 \mathrm{H}), 0.88(\mathrm{~s}, 9 \mathrm{H}), 0.09(\mathrm{~s}, 3 \mathrm{H}), 0.06(\mathrm{~s}, 3 \mathrm{H}) .{ }^{13} \mathrm{C}\left\{{ }^{1} \mathrm{H}\right\}$ NMR $\left(\mathrm{CD}_{3} \mathrm{OD}, 150 \mathrm{MHz}\right): \delta 173.7,170.5,138.0,137.9,130.9$, $130.8,129.7,109.9,88.4,77.1,76.8,71.1,70.7,68.0,53.3,52.6,42.8$, 27.0, 26.1, 25.7, 23.0, 18.7, -4.3, -4.7. HRMS (ESI-TOF) $\mathrm{m} / z$ : [M + $\mathrm{H}]^{+}$calcd for $\mathrm{C}_{27} \mathrm{H}_{44} \mathrm{NO}_{8} \mathrm{SSi}$, 570.2551; found, 570.2577. 
Methyl (Methyl 5-acetamido-7,8,9-tri-O-acetyl-4-O-(tert-butyldimethylsilyl)-3,5-dideoxy-D-glycero- $\alpha$-D-galacto- 2 nonulopyranosid)onate (11). To a solution of $7(3.45 \mathrm{~g}, 7 \mathrm{mmol})$ in dichloromethane $(130 \mathrm{~mL})$ was added $50 \%$ aqueous TFA $(5.1 \mathrm{~mL}$, $33.3 \mathrm{mmol}, 4.75$ equiv). The reaction was stirred at room temperature for $1 \mathrm{~h}$ (monitored by TLC/EtOAc/ $\mathrm{CH}_{3} \mathrm{OH} / \mathrm{H}_{2} \mathrm{O}(10: 2: 1, \mathrm{v} / \mathrm{v} / \mathrm{v})$ ). After completion, triethylamine $(3 \mathrm{~mL})$ was added to the mixture, and the solvents were concentrated to dryness. The residue was coevaporated three times with toluene and directly acetylated. Crude compound 9 was dissolved in pyridine $(35 \mathrm{~mL})$ and treated with an excess of acetic anhydride ( $10 \mathrm{~mL}, 105 \mathrm{mmol}, 15$ equiv). The reaction was allowed to perform at room temperature and under a nitrogen atmosphere overnight. After completion, the mixture was coevaporated three times with toluene. The residual oil was purified using flash chromatography (petroleum ether/EtOAc, gradient 5$70 \%)$ to give $11(3.2 \mathrm{~g}, 79 \%)$ as an off-white solid. ${ }^{1} \mathrm{H}$ NMR $\left(\mathrm{CDCl}_{3}\right.$, $600 \mathrm{MHz}$ ): $\delta 5.36(\mathrm{ddd}, 1 \mathrm{H}, J=8.8,5.7,2.8 \mathrm{~Hz}), 5.29(\mathrm{dd}, 1 \mathrm{H}, J=$ 8.7, $2.3 \mathrm{~Hz}$ ), 4.29 (dd, $1 \mathrm{H}, J=12.4,2.8 \mathrm{~Hz}), 4.03(\mathrm{dd}, 1 \mathrm{H}, J=12.4$, $5.7 \mathrm{~Hz}), 3.97(\mathrm{dd}, 1 \mathrm{H}, J=11.0,2.1 \mathrm{~Hz}), 3.81-3.72(\mathrm{~m}, 1 \mathrm{H}), 3.78(\mathrm{~s}$, $3 \mathrm{H}), 3.52-3.44(\mathrm{~m}, 1 \mathrm{H}), 3.24(\mathrm{~s}, 3 \mathrm{H}), 2.49(\mathrm{dd}, 1 \mathrm{H}, J=12.8,4.32$ $\mathrm{Hz}), 2.10(\mathrm{~s}, 3 \mathrm{H}), 2.07(\mathrm{~s}, 3 \mathrm{H}), 1.97(\mathrm{~s}, 3 \mathrm{H}), 1.83(\mathrm{~s}, 3 \mathrm{H}), 1.65(\mathrm{dd}$, $1 \mathrm{H}, J=12.85,11.83 \mathrm{~Hz}), 0.83(\mathrm{~s}, 9 \mathrm{H}), 0.09(\mathrm{~s}, 3 \mathrm{H}), 0.00(\mathrm{~s}, 3 \mathrm{H})$. ${ }^{13} \mathrm{C}\left\{{ }^{1} \mathrm{H}\right\}$ NMR $\left(\mathrm{CDCl}_{3}, 150 \mathrm{MHz}\right): \delta 173.1,172.4,171.8,171.7$, 169.6, 100.4, 73.3, 70.6, 69.8, 68.9, 63.6, 53.0, 52.6, 52.5, 43.0, 26.1, 23.1, 21.2, 20.9, 20.6, 18.7, -4.5, -4.8. HRMS (ESI-TOF) $\mathrm{m} / \mathrm{z}$ : [M+ $\mathrm{Na}]^{+}$calcd for $\mathrm{C}_{25} \mathrm{H}_{43} \mathrm{NO}_{12} \mathrm{SiNa}$, 600.2447; found, 600.2471.

Methyl (Phenyl 5-acetamido-7,8,9-tri-O-acetyl-4-O-(tert-butyldimethylsilyl)-3,5-dideoxy-2-thio-D-glycero- $\alpha$-D-galacto-2nonulopyranosid)onate (12). To a solution of 8 (650 mg, 1.14 $\mathrm{mmol})$ in dichloromethane $(20 \mathrm{~mL})$ was added $50 \%$ aqueous TFA $(0.83 \mathrm{~mL}, 5.4 \mathrm{mmol}, 4.75$ equiv). The reaction was stirred at room temperature for $30 \mathrm{~min}$ (monitored by TLC/EtOAc/ $/ \mathrm{CH}_{3} \mathrm{OH} / \mathrm{H}_{2} \mathrm{O}$ $(10: 2: 1, \mathrm{v} / \mathrm{v} / \mathrm{v}))$. After completion, triethylamine $(0.5 \mathrm{~mL})$ was then added to the mixture, and the solvents were concentrated to dryness. The residue was coevaporated three times with toluene and directly acetylated. Crude compound $\mathbf{1 0}$ was dissolved in pyridine $(7 \mathrm{~mL})$ and treated with an excess of acetic anhydride $(1.6 \mathrm{~mL}, 17 \mathrm{mmol}, 15$ equiv). The reaction was allowed to perform at room temperature and under a nitrogen atmosphere overnight. After completion, the mixture was coevaporated three times with toluene. The residual oil was purified using flash chromatography (petroleum ether/EtOAc, gradient $5-70 \%)$ to give $12(605 \mathrm{mg}, 81 \%)$ as a pale-yellow solid. ${ }^{1} \mathrm{H}$ NMR $\left(\mathrm{CDCl}_{3}, 600 \mathrm{MHz}\right): \delta 7.50(\mathrm{dd}, 2 \mathrm{H}, J=8.2,1.4 \mathrm{~Hz}), 7.37$ $(\mathrm{d}, 1 \mathrm{H}, J=7.0 \mathrm{~Hz}), 7.32(\mathrm{t}, 2 \mathrm{H}, J=7.5 \mathrm{~Hz}), 5.29(\mathrm{dd}, 1 \mathrm{H}, J=7.3,1.8$ $\mathrm{Hz}$ ), 5.25 (ddd, $1 \mathrm{H}, J=7.1,5.4,2.6 \mathrm{~Hz}), 5.18(\mathrm{~d}, 1 \mathrm{H}, J=9.1 \mathrm{~Hz})$, $4.38(\mathrm{dd}, 1 \mathrm{H}, J=12.5,2.6 \mathrm{~Hz}), 4.25(\mathrm{dd}, 1 \mathrm{H}, J=12.5,5.4 \mathrm{~Hz}), 4.06$ $(\mathrm{dd}, 1 \mathrm{H}, J=10.8,1.8 \mathrm{~Hz}), 3.88(\mathrm{dt}, 1 \mathrm{H}, J=10.3,4.4 \mathrm{~Hz}), 3.53(\mathrm{~s}$, $3 \mathrm{H}), 3.37(\mathrm{q}, 1 \mathrm{H}, J=9.6 \mathrm{~Hz}), 2.78(\mathrm{dd}, 1 \mathrm{H}, J=13.0,4.5 \mathrm{~Hz}), 2.17(\mathrm{~s}$, $3 \mathrm{H}), 2.05(\mathrm{~s}, 3 \mathrm{H}), 2.02(\mathrm{~s}, 3 \mathrm{H}), 1.91(\mathrm{~s}, 3 \mathrm{H}), 1.84(\mathrm{dd}, 1 \mathrm{H}, J=12.9$, $11.2 \mathrm{~Hz}), 0.85(\mathrm{~s}, 9 \mathrm{H}), 0.05(\mathrm{~s}, 3 \mathrm{H}), 0.01(\mathrm{~s}, 3 \mathrm{H}) .{ }^{13} \mathrm{C}\left\{{ }^{1} \mathrm{H}\right\} \mathrm{NMR}$ $\left(\mathrm{CDCl}_{3}, 150 \mathrm{MHz}\right): \delta 136.4,129.8,129.2,128.9,87.7,73.7,70.1$, 68.6, 68.5, 62.2, 53.5, 52.5, 42.4, 25.7, 23.8, 21.1, 21.0, 18.0, -4.4, -4.8. HRMS (ESI-TOF) $\mathrm{m} / z$ : $[\mathrm{M}+\mathrm{H}]^{+}$calcd for $\mathrm{C}_{30} \mathrm{H}_{46} \mathrm{NO}_{11} \mathrm{SSi}$, 656.2555; found, 656.2584 .

Methyl (Methyl 5-acetamido-7,8,9-tri-O-acetyl-3,5-dideoxy-Dglycero- $\alpha$-D-galacto-2-nonulopyranosid)onate (6). Compound 11 $(3.2 \mathrm{~g}, 5.54 \mathrm{mmol})$ was dissolved in dry THF $(73 \mathrm{~mL})$, and the mixture was stirred at room temperature under a nitrogen atmosphere. A $1 \mathrm{M}$ TBAF solution in THF $(11 \mathrm{~mL}, 11 \mathrm{mmol}, 2$ equiv) was then added to the reaction. After $1.5 \mathrm{~h}$, the reaction was complete, and the solvents were concentrated to dryness. The residue was purified using flash chromatography $\left(\mathrm{CH}_{2} \mathrm{Cl}_{2} / \mathrm{CH}_{3} \mathrm{OH}\right.$, gradient $0-8 \%)$ to give $6(2.07 \mathrm{~g}, 81 \%)$ as a white solid. ${ }^{1} \mathrm{H}$ NMR $\left(\mathrm{CD}_{3} \mathrm{OD}\right.$, $600 \mathrm{MHz}): \delta 5.40(\mathrm{ddd}, 1 \mathrm{H}, J=8.7,5.8,2.8 \mathrm{~Hz}), 5.30(\mathrm{dd}, 1 \mathrm{H}, J=$ 8.6, $2.3 \mathrm{~Hz}$ ), $4.33(\mathrm{dd}, 1 \mathrm{H}, J=12.4,2.8 \mathrm{~Hz}), 4.06(\mathrm{dd}, 1 \mathrm{H}, J=12.4$, $5.7 \mathrm{~Hz}), 4.03(\mathrm{dd}, 1 \mathrm{H}, J=10.7,2.3 \mathrm{~Hz}), 3.80(\mathrm{~s}, 3 \mathrm{H}), 3.75(\mathrm{t}, 1 \mathrm{H}, J=$ $10.3 \mathrm{~Hz}$ ), 3.41 (ddd, $1 \mathrm{H}, J=12.2,10.0,4.4 \mathrm{~Hz}), 3.27(\mathrm{~s}, 3 \mathrm{H}), 2.56$ $(\mathrm{dd}, 1 \mathrm{H}, J=12.9,4.4 \mathrm{~Hz}), 2.13(\mathrm{~s}, 3 \mathrm{H}), 2.10(\mathrm{~s}, 3 \mathrm{H}), 2.01(\mathrm{~s}, 3 \mathrm{H})$, $1.90(\mathrm{~s}, 3 \mathrm{H}), 1.67$ (appt, $1 \mathrm{H}, J=12.5 \mathrm{~Hz}) .{ }^{13} \mathrm{C}\left\{{ }^{1} \mathrm{H}\right\} \mathrm{NMR}\left(\mathrm{CD}_{3} \mathrm{OD}\right.$, $150 \mathrm{MHz}$ ): $\delta 173.8,172.4,171.8,171.7,169.8,100.6,73.4,69.8,69.2$,
69.0, 63.6, 53.0, 52.8, 52.5, 42.0, 22.9, 21.2, 20.9, 20.6. HRMS (ESITOF) $m / z:[\mathrm{M}+\mathrm{Na}]^{+}$calcd for $\mathrm{C}_{19} \mathrm{H}_{29} \mathrm{NO}_{12} \mathrm{Na}$, 486.1582; found, 486.1604

Methyl (Phenyl 5-acetamido-7,8,9-tri-O-acetyl-3,5-dideoxy-2thio-D-glycero- $\alpha$-D-galacto-2-nonulopyranosid)onate (13). Compound $12(590 \mathrm{mg}, 0.90 \mathrm{mmol})$ was dissolved in dry THF (12 $\mathrm{mL}$ ), and the mixture was stirred at room temperature under a nitrogen atmosphere. A $1 \mathrm{M}$ TBAF solution in THF $(1.8 \mathrm{~mL}, 1.8$ mmol, 2 equiv) was then added to the reaction. After $50 \mathrm{~min}$, the reaction was complete, and the solvents were concentrated to dryness. The residue was purified using flash chromatography $\left(\mathrm{Tol} / \mathrm{CH}_{3} \mathrm{OH}\right.$, $5-12 \%)$ to give $13(310 \mathrm{mg}, 64 \%)$ as a pale-yellow solid. ${ }^{1} \mathrm{H}$ NMR $\left(\mathrm{CD}_{3} \mathrm{OD}, 600 \mathrm{MHz}\right): \delta 7.52(\mathrm{dd}, 2 \mathrm{H}, J=8.0,1.4 \mathrm{~Hz}), 7.40(\mathrm{~d}, 1 \mathrm{H}, J$ $=7.4 \mathrm{~Hz}), 7.35(\mathrm{t}, 2 \mathrm{H}, J=7.5 \mathrm{~Hz}), 5.29(\mathrm{dd}, 1 \mathrm{H}, J=7.5,1.9 \mathrm{~Hz})$, $5.26(\mathrm{ddd}, 1 \mathrm{H}, J=7.7,5.3,2.6 \mathrm{~Hz}), 4.40(\mathrm{dd}, 1 \mathrm{H}, J=12.4,2.6 \mathrm{~Hz})$, $4.14(\mathrm{dd}, 1 \mathrm{H}, J=12.4,5.3 \mathrm{~Hz}$ ), $3.83(\mathrm{dd}, 1 \mathrm{H}, J=10.7,1.8 \mathrm{~Hz}), 3.70$ $(\mathrm{t}, 1 \mathrm{H}, J=10.3 \mathrm{~Hz}), 3.53(\mathrm{~s}, 3 \mathrm{H}), 3.41(\mathrm{ddd}, 1 \mathrm{H}, J=11.4,10.0,4.6$ $\mathrm{Hz}), 2.80(\mathrm{dd}, 1 \mathrm{H}, J=13.0,4.5 \mathrm{~Hz}), 2.12(\mathrm{~s}, 3 \mathrm{H}), 2.03(\mathrm{~s}, 3 \mathrm{H}), 2.01$ $(\mathrm{s}, 3 \mathrm{H}), 1.89(\mathrm{~s}, 3 \mathrm{H}), 1.80(\mathrm{dd}, 1 \mathrm{H}, J=13.0,11.5 \mathrm{~Hz}) .{ }^{13} \mathrm{C}\left\{{ }^{1} \mathrm{H}\right\}$ NMR $\left(\mathrm{CD}_{3} \mathrm{OD}, 150 \mathrm{MHz}\right): \delta 173.8,172.5,171.7,171.6,169.7$, 137.4, 130.8, 130.6, 130.0, 89.1, 75.7, 71.1, 69.8, 69.3, 63.2, 53.0, 52.6, 42.4, 22.9, 21.0, 20.9, 20.7. HRMS (ESI-TOF) $\mathrm{m} / z:[\mathrm{M}+\mathrm{H}]^{+}$calcd for $\mathrm{C}_{24} \mathrm{H}_{32} \mathrm{NO}_{11} \mathrm{~S}$, 542.1691; found, 542.1717.

Methyl (Methyl 5-(N-tert-butoxycarbonylacetamido)-7,8,9-tri-Oacetyl-4-O-(tert-butyldimethylsilyl)-3,5-dideoxy-D-glycero- $\alpha$-D-galacto-2-nonulopyranosid)onate (14). Compound 11 (500 mg, 0.87 $\mathrm{mmol})$, di-tert-butyl dicarbonate ( $567 \mathrm{mg}, 2.6 \mathrm{mmol}, 3$ equiv), and DMAP $(63.4 \mathrm{mg}, 0.52 \mathrm{mmol}, 0.6$ equiv) were charged in a roundbottom flask, and dry THF $(14 \mathrm{~mL})$ was added. The mixture was heated to reflux temperature for $8 \mathrm{~h}$ (monitored by TLC: petroleum ether/EtOAc $\left.(3: 2, \mathrm{v} / \mathrm{v}), R_{f}=0.50\right)$. The mixture was then diluted in diethyl ether $(40 \mathrm{~mL})$, washed with $0.5 \mathrm{M} \mathrm{HCl}(\mathrm{aq}, 15 \mathrm{~mL})$, and sat. $\mathrm{NaHCO}_{3}(\mathrm{aq}, 15 \mathrm{~mL})$. The organic layer was dried over anhydrous sodium sulfate, filtered, and concentrated to dryness. The residue was purified using flash chromatography (petroleum ether/EtOAc, gradient $5-45 \%)$ to give 14 (400 mg, 68\%) as a white solid. In ${ }^{1} \mathrm{H}$ and ${ }^{13} \mathrm{C}$ NMR experiments, a splitting of the signals is observed due to the presence of $N$-Boc rotamers (2:5 ratio). Chemical shifts are only reported for the main rotamer. ${ }^{1} \mathrm{H} \mathrm{NMR}\left(\mathrm{CD}_{3} \mathrm{OD}, 600 \mathrm{MHz}\right): \delta$ $3.97-3.73(\mathrm{~m}, 8 \mathrm{H}), 3.70-3.55(\mathrm{~m}, 12 \mathrm{H}), 3.50(\mathrm{dd}, 1 \mathrm{H}, J=6.6,1.7$ $\mathrm{Hz}), 3.38(\mathrm{t}, 2 \mathrm{H}, J=4.9 \mathrm{~Hz}), 2.70(\mathrm{dd}, 1 \mathrm{H}, J=12.8,4.6 \mathrm{~Hz}), 2.00(\mathrm{~s}$, $3 \mathrm{H}), 1.75(\mathrm{t}, 1 \mathrm{H}, J=12.3 \mathrm{~Hz}) .{ }^{13} \mathrm{C}\left\{{ }^{1} \mathrm{H}\right\}$ NMR $\left(\mathrm{CD}_{3} \mathrm{OD}, 150 \mathrm{MHz}\right)$ : $\delta 175.2,172.4,171.8,171.6,169.5,153.9,100.4,85.9,71.8,69.8,68.2$, 67.6, 62.9, 56.4, 53.2, 52.5, 44.4, 28.4, 28.3, 27.3, 26.3, 26.0, 21.4, 21.0, 20.6, -4.1, -4.6. HRMS (ESI-TOF) $\mathrm{m} / \mathrm{z}:[\mathrm{M}+\mathrm{Na}]^{+}$calcd for $\mathrm{C}_{30} \mathrm{H}_{51} \mathrm{NO}_{14} \mathrm{SiNa}$, 700.2971; found, 700.3003 .

Methyl (Methyl 5-(N-tert-butoxycarbonylacetamido)-7,8,9-tri-Oacetyl-3,5-dideoxy-D-glycero- $\alpha$-D-galacto-2-nonulopyranosid)onate (15). Compound 14 (400 $\mathrm{mg}, 0.59 \mathrm{mmol})$ was dissolved in dry THF $(7.7 \mathrm{~mL})$, and the mixture was stirred at room temperature under a nitrogen atmosphere. A $1 \mathrm{M}$ TBAF solution in THF $(1.2 \mathrm{~mL}$, $1.2 \mathrm{mmol}, 2$ equiv) was then added to the reaction. After $1 \mathrm{~h}$, the reaction was complete, and the solvents were concentrated to dryness. The residue was purified using flash chromatography $\left(\mathrm{Tol} / \mathrm{CH}_{3} \mathrm{OH}\right.$, 4-10\%) to give $15(172 \mathrm{mg}, 52 \%)$ as a white solid. ${ }^{1} \mathrm{H} \mathrm{NMR}\left(\mathrm{CDCl}_{3}\right.$, $600 \mathrm{MHz}): \delta 5.48-5.40(\mathrm{~m}, 2 \mathrm{H}), 4.78(\mathrm{ddd}, 1 \mathrm{H}, J=12.3,10.4,4.6$ $\mathrm{Hz}), 4.29(\mathrm{dd}, 1 \mathrm{H}, J=12.6,2.4 \mathrm{~Hz}), 4.19(\mathrm{~d}, 1 \mathrm{H}, J=10.3 \mathrm{~Hz}), 4.10$ $(\mathrm{dd}, 1 \mathrm{H}, J=12.4,4.5 \mathrm{~Hz}), 4.04(\mathrm{dd}, 1 \mathrm{H}, J=11.0,2.0 \mathrm{~Hz}), 3.80(\mathrm{~s}$, $3 \mathrm{H}), 3.78(\mathrm{q}, 1 \mathrm{H}, J=10.6 \mathrm{~Hz}), 3.31(\mathrm{~s}, 3 \mathrm{H}), 2.58(\mathrm{dd}, 1 \mathrm{H}, J=12.8$, $4.6 \mathrm{~Hz}), 2.14(\mathrm{~s}, 3 \mathrm{H}), 2.13(\mathrm{~s}, 3 \mathrm{H}), 2.04(\mathrm{~s}, 3 \mathrm{H}), 2.03(\mathrm{~s}, 3 \mathrm{H}), 1.90$ (appt, $1 \mathrm{H}, J=12.7 \mathrm{~Hz}), 1.39(\mathrm{~s}, 9 \mathrm{H}) .{ }^{13} \mathrm{C}\left\{{ }^{1} \mathrm{H}\right\}$ NMR $\left(\mathrm{CDCl}_{3}, 150\right.$ $\mathrm{MHz}): \delta 170.9,170.8,170.1,169.9,168.3,155.3,99.1,80.3,72.8$ 69.5, 68.3, 67.5, 62.7, 52.9, 52.6, 50.8, 38.2, 28.3, 21.3, 21.0. HRMS (ESI-TOF) $\mathrm{m} / z$ : $[\mathrm{M}+\mathrm{Na}]^{+}$calcd for $\mathrm{C}_{24} \mathrm{H}_{37} \mathrm{NO}_{14} \mathrm{Na}$, 586.2106; found, 586.2130.

Methyl (Methyl 5-acetamido-7,8,9-tri-O-acetyl-3,5-dideoxy-4propargyloxy-D-glycero- $\alpha$-D-galacto-2-nonulopyranosid)onate (16). Compound $17(30 \mathrm{mg}, 0.08 \mathrm{mmol})$ was dissolved in pyridine $(1.3 \mathrm{~mL})$ and treated with an excess of acetic anhydride $(0.15 \mathrm{~mL}, 1.6$ mmol, 20 equiv). The reaction was allowed to perform at room 
temperature and under a nitrogen atmosphere overnight. The mixture was diluted in EtOAc $(10 \mathrm{~mL})$, washed with $1 \mathrm{M} \mathrm{HCl}(\mathrm{aq}, 5 \mathrm{~mL})$ twice, and then with brine $(5 \mathrm{~mL})$. The organic layer was dried over anhydrous sodium sulfate, filtered, and concentrated to dryness to give semipure $16(24 \mathrm{mg}, 60 \%)$ as a colorless film. Analytical samples were obtained after purification of the semipure product using reverse-phase $\mathrm{HPLC}\left(\mathrm{H}_{2} \mathrm{O} /\right.$ acetonitrile $(0.005 \%$ formic acid), gradient $15-80 \%$ over $25 \mathrm{~min}) .{ }^{1} \mathrm{H}$ NMR $\left(\mathrm{CDCl}_{3}, 600 \mathrm{MHz}\right): \delta$ $5.44(\mathrm{ddd}, 1 \mathrm{H}, J=8.5,5.5,2.7 \mathrm{~Hz}), 5.39-5.26(\mathrm{~m}, 2 \mathrm{H}), 4.33(\mathrm{dd}$, 1H. $J=12.5,2.6 \mathrm{~Hz}), 4.27(\mathrm{dd}, 1 \mathrm{H}, J=10.7,2.1 \mathrm{~Hz}), 4.23(\mathrm{dd}, 1 \mathrm{H}, J$ $=16.1,2.3 \mathrm{~Hz}), 4.16(\mathrm{dd}, 1 \mathrm{H}, J=12.5,5.5 \mathrm{~Hz}), 4.13(\mathrm{dd}, 1 \mathrm{H}, J=$ 16.0, $2.3 \mathrm{~Hz}), 3.88(\mathrm{dd}, 1 \mathrm{H}, J=11.8,9.7,4.5 \mathrm{~Hz}), 3.82(\mathrm{~s}, 3 \mathrm{H}), 3.49$ $(\mathrm{t}, 1 \mathrm{H}, J=2.4 \mathrm{~Hz}), 3.32(\mathrm{~s}, 3 \mathrm{H}), 2.76(\mathrm{dd}, 1 \mathrm{H}, J=12.8,4.5 \mathrm{~Hz}), 2.42$ $(\mathrm{t}, 1 \mathrm{H}, J=2.4 \mathrm{~Hz}), 2.15(\mathrm{~s}, 3 \mathrm{H}), 2.14(\mathrm{~s}, 3 \mathrm{H}), 2.04(\mathrm{~s}, 3 \mathrm{H}), 1.97(\mathrm{~s}$, $3 \mathrm{H}), 1.68(\mathrm{dd}, 1 \mathrm{H}, J=12.8,11.9 \mathrm{~Hz}) .{ }^{13} \mathrm{C}\left\{{ }^{1} \mathrm{H}\right\} \operatorname{NMR}\left(\mathrm{CDCl}_{3}, 150\right.$ $\mathrm{MHz}): \delta 170.8,170.7,170.2,168.2,99.3,79.8,74.7,73,1,71.5,68.6$, 68.0, 62.6, 56.6, 52.7, 52.5, 51.4, 37.9, 23.9, 21.3, 21.1, 20.9. HRMS (ESI-TOF) $m / z:[\mathrm{M}+\mathrm{Na}]^{+}$calcd for $\mathrm{C}_{22} \mathrm{H}_{31} \mathrm{NO}_{12} \mathrm{Na}, 524.1738$; found, 524.1756 .

Methyl (Methyl 5-acetamido-3,5-dideoxy-4-propargyloxy-Dglycero- $\alpha$-D-galacto-2-nonulopyranosid)onate (17). Compound 17 was synthesized starting from $6(40 \mathrm{mg}, 0.086 \mathrm{mmol})$ using the general procedure for $O$-alkylation $\left(\mathrm{GP} 1 ; \mathrm{TLC} / \mathrm{EtOAc} ; R_{f}=0.43\right.$ ) and the general procedure for deacetylation (GP2; TLC: Tol/ $\left.\mathrm{CH}_{3} \mathrm{OH}(4: 1, \mathrm{v} / \mathrm{v}) ; R_{f}=0.40\right)$, successively. The compound was purified using flash chromatography $\left(\mathrm{Tol} / \mathrm{CH}_{3} \mathrm{OH}\right.$, gradient $\left.5-20 \%\right)$ to give 17 as a white solid and in $70 \%$ yield. ${ }^{1} \mathrm{H}$ NMR $\left(\mathrm{CD}_{3} \mathrm{OD}, 600\right.$ $\mathrm{MHz}): \delta 4.27-4.19(\mathrm{~m}, 2 \mathrm{H}), 3.90-3.80(\mathrm{~m}, 3 \mathrm{H}), 3.85(\mathrm{~s}, 3 \mathrm{H})$, $3.74-3.62(\mathrm{~m}, 3 \mathrm{H}), 3.51(\mathrm{dd}, 1 \mathrm{H}, J=9.0,1.6 \mathrm{~Hz}), 3.35(\mathrm{~s}, 3 \mathrm{H}), 2.91$ $(\mathrm{t}, 1 \mathrm{H}, J=2.5 \mathrm{~Hz}), 2.86(\mathrm{dd}, 1 \mathrm{H}, J=12.9,4.6 \mathrm{~Hz}), 1.98(\mathrm{~s}, 3 \mathrm{H}), 1.65$ $(\mathrm{dd}, 1 \mathrm{H}, J=12.9,11.7 \mathrm{~Hz}) .{ }^{13} \mathrm{C}\left\{{ }^{1} \mathrm{H}\right\} \mathrm{NMR}\left(\mathrm{CD}_{3} \mathrm{OD}, 150 \mathrm{MHz}\right): \delta$ 174.7, 170.7, 100.3, 80.7, 76.1, 75.8, 74.6, 72.4, 70.1, 64.7, 57.6, 53.3, $52.0,51.9,38.5,22.7$. HRMS (ESI-TOF) $\mathrm{m} / z:[\mathrm{M}+\mathrm{Na}]^{+}$calcd for $\mathrm{C}_{16} \mathrm{H}_{25} \mathrm{NO}_{9} \mathrm{Na}$, 398.1422; found, 398.1438 .

Methyl (Methyl 5-acetamido-9-O-benzyl-3,5-dideoxy-D-glycero$\alpha$-D-galacto-2-nonulopyranosid)onate (20). ${ }^{3}$ Compound 20 was synthesized in a quantitative yield following the procedure described in ref 3. ${ }^{1} \mathrm{H}$ NMR $\left(\mathrm{CD}_{3} \mathrm{OD}, 600 \mathrm{MHz}\right): \delta 7.36(\mathrm{~d}, 1 \mathrm{H} \mathrm{J}=7.6 \mathrm{~Hz})$, $7.33(\mathrm{t}, 2 \mathrm{H}, J=7.3 \mathrm{~Hz}), 7.26(\mathrm{t}, 2 \mathrm{H}, J=7.2 \mathrm{~Hz}), 4.59(\mathrm{ABq}, 2 \mathrm{H}, J=$ $12.2 \mathrm{~Hz}), 3.99(\mathrm{ddd}, 1 \mathrm{H}, J=9.1,5.9,2.3 \mathrm{~Hz}), 3.83(\mathrm{~s}, 3 \mathrm{H}), 3.79(\mathrm{dd}$, $1 \mathrm{H} J=10.4,2.4 \mathrm{~Hz}), 3.76(\mathrm{t}, 1 \mathrm{H}, J=10.3 \mathrm{~Hz}), 3.68-3.61(\mathrm{~m}, 2 \mathrm{H})$, $3.61-3.56(\mathrm{~m}, 2 \mathrm{H}), 3.32(\mathrm{~s}, 3 \mathrm{H}), 2.65(\mathrm{dd}, 1 \mathrm{H}, J=12.9,4.6 \mathrm{~Hz})$, $1.99(\mathrm{~s}, 3 \mathrm{H}), 1.71(\mathrm{dd}, 1 \mathrm{H}, J=12.9,11.9 \mathrm{~Hz}) .{ }^{13} \mathrm{C}\left\{{ }^{1} \mathrm{H}\right\} \mathrm{NMR}$ $\left(\mathrm{CD}_{3} \mathrm{OD}, 150 \mathrm{MHz}\right): \delta 175.1,170.8,139.8,129.3,128.8,128.6$, 100.3, 74.7, 74.4, 73.0, 71.4, 70.1, 68.5, 53.8, 53.3, 52.0, 41.4, 22.7. HRMS (ESI-TOF) $m / z:[\mathrm{M}+\mathrm{Na}]^{+}$calcd for $\mathrm{C}_{20} \mathrm{H}_{29} \mathrm{NO}_{9} \mathrm{Na}$, 450.1735; found, 450.1743 .

Methyl (Methyl 5-acetamido-9-benzyloxy-3,5-dideoxy-7,8-O-isopropylidene-D-glycero- $\alpha$-D-galacto-2-nonulopyranosid)onate (18). Compound $20(307 \mathrm{mg}, 0.72 \mathrm{mmol})$ and $(1 R)-(-)$-10-camphorsulfonic acid ( $17 \mathrm{mg}, 0.072 \mathrm{mmol}, 0.1$ equiv) were dissolved in freshly distilled acetonitrile $(1.45 \mathrm{~mL})$. The stirring mixture was cooled to an ice bath temperature, and 2,2-dimethoxypropane $(0.26 \mathrm{~mL}, 2.15$ mmol, 3.0 equiv) was added under a nitrogen atmosphere. The reaction mixture was then allowed to warm to room temperature, and after $4 \mathrm{~h}$, triethylamine was added. The volatiles were removed under a vacuum, and the residue was purified using flash chromatography (Tol/acetone, isocratic 7:3) to afford $18(323 \mathrm{mg}, 96 \%) .{ }^{1} \mathrm{H}$ NMR $\left(\mathrm{CD}_{3} \mathrm{OD}, 600 \mathrm{MHz}\right): \delta 7.38(\mathrm{~d}, 2 \mathrm{H}, J=7.4 \mathrm{~Hz}), 7.32(\mathrm{t}, 2 \mathrm{H}, J=7.4$ $\mathrm{Hz}), 7.26(\mathrm{t}, 1 \mathrm{H}, J=7.3 \mathrm{~Hz}), 4.63(\mathrm{ABq}, 2 \mathrm{H}, J=12.3 \mathrm{~Hz}), 4.50(\mathrm{td}$, $1 \mathrm{H}, J=7.1,4.3 \mathrm{~Hz}), 4.17(\mathrm{~d}, 1 \mathrm{H}, J=6.9 \mathrm{~Hz}), 4.02(\mathrm{dd}, 1 \mathrm{H}, J=10.4$, $7.6 \mathrm{~Hz}), 3.96(\mathrm{dd}, 1 \mathrm{H}, J=10.5,4.3 \mathrm{~Hz}), 3.84-3.78(\mathrm{~m}, 1 \mathrm{H}), 3.50$ (ddd, $1 \mathrm{H}, J=12.4,10.0,4.3 \mathrm{~Hz}), 2.52(\mathrm{dd}, 1 \mathrm{H}, J=12.6,4.4 \mathrm{~Hz}), 1.94$ $(\mathrm{s}, 3 \mathrm{H}), 1.59($ appt, $1 \mathrm{H}, J=12.5 \mathrm{~Hz}), 1.48(\mathrm{~s}, 3 \mathrm{H}), 1.34(\mathrm{~s}, 3 \mathrm{H})$. ${ }^{13} \mathrm{C}\left\{{ }^{1} \mathrm{H}\right\}$ NMR $\left(\mathrm{CD}_{3} \mathrm{OD}, 150 \mathrm{MHz}\right): \delta 173.8,170.3,139.6,129.4$, $129.1,128.7,110.4,100.5,77.8,76.0,74.2,74.1,70.2,68.4,54.2,52.8$, 52.3, 41.7, 26.7, 26.0, 23.0. HRMS (ESI-TOF) $\mathrm{m} / z:[\mathrm{M}+\mathrm{Na}]^{+}$calcd for $\mathrm{C}_{23} \mathrm{H}_{33} \mathrm{NO}_{9} \mathrm{Na}$, 490.2048; found, 490.2062.

Methyl (Methyl 5-acetamido-9-benzyloxy-3,5-dideoxy-7,8-0-isopropylidene-4-propargyloxy-D-glycero- $\alpha$-D-galacto-2- nonulopyranosid)onate (22). Compound 22 was synthesized starting from $18(40.3 \mathrm{mg}, 0.086 \mathrm{mmol})$ using the general procedure for $O$-alkylation (GP1) with 1.1 equiv of $\mathrm{NaH}$ and 5 equiv of propargyl bromide (TLC: petroleum ether/EtOAc $(1: 4, \mathrm{v} / \mathrm{v}) ; R_{f}=$ $0.2)$. The compound was purified using flash chromatography (petroleum ether/EtOAc, gradient 30-90\%) to give $22(24.9 \mathrm{mg}$, $57 \%)$ as a white solid. ${ }^{1} \mathrm{H}$ NMR $\left(\mathrm{CD}_{3} \mathrm{OD}, 600 \mathrm{MHz}\right): \delta 7.38(\mathrm{~d}, 2 \mathrm{H}, J$ $=7.5 \mathrm{~Hz}), 7.33(\mathrm{t}, 2 \mathrm{H}, J=7.6 \mathrm{~Hz}), 7.27(\mathrm{t}, 1 \mathrm{H}, J=7.3 \mathrm{~Hz}), 4.63$ $(\mathrm{ABq}, 2 \mathrm{H}, J=12.1 \mathrm{~Hz}), 4.50(\mathrm{dt}, 1 \mathrm{H}, J=7.2,4.4,1 \mathrm{H}), 4.19-4.15(\mathrm{~m}$, $3 \mathrm{H}), 4.01(\mathrm{dd}, 1 \mathrm{H}, J=10.4,7.5 \mathrm{~Hz}), 3.96(\mathrm{dd}, 1 \mathrm{H}, J=10.3,4.5 \mathrm{~Hz})$, $3.86(\mathrm{t}, 1 \mathrm{H}, J=10.2 \mathrm{~Hz}), 3.79(\mathrm{~s}, 3 \mathrm{H}), 3.78(\mathrm{~d}, 1 \mathrm{H}, J=6.9 \mathrm{~Hz}), 3.61$ $(\mathrm{ddd}, 1 \mathrm{H}, J=12.1,10.1,4.3 \mathrm{~Hz}), 3.20(\mathrm{~s}, 3 \mathrm{H}), 2.86(\mathrm{t}, 1 \mathrm{H}, J=2.4$ $\mathrm{Hz}), 2.73(\mathrm{dd}, 1 \mathrm{H}, J=12.6,4.3 \mathrm{~Hz}), 1.92(\mathrm{~s}, 3 \mathrm{H}), 1.50(\mathrm{dd}, 1 \mathrm{H}, J=$ 12.9, $12.0 \mathrm{~Hz}), 1.47(\mathrm{~s}, 3 \mathrm{H}), 1.34(\mathrm{~s}, 3 \mathrm{H}) .{ }^{13} \mathrm{C}\left\{{ }^{1} \mathrm{H}\right\}$ NMR $\left(\mathrm{CD}_{3} \mathrm{OD}\right.$, $150 \mathrm{MHz}): \delta 177.2,173.5,170.1,139.6,129.3,129.1,128.7,110.5$, $100.5,80.7,77.7,76.0,75.9,75.1,74.2,74.0,70.2,57.4,52.9,52.4$, 52.3, 38.9, 26.7, 25.9, 23.0. HRMS (ESI-TOF) $m / z:[\mathrm{M}+\mathrm{Na}]^{+}$calcd for $\mathrm{C}_{26} \mathrm{H}_{35} \mathrm{NO}_{9} \mathrm{Na}$, 528.2204; found, 528.2218.

Methyl (Phenyl 5-acetamido-3,5-dideoxy-4-propargyloxy-2thio- $D$-glycero- $\alpha$-D-galacto-2-nonulopyranosid)onate (23). Compound 23 was synthesized starting from $13(46.7 \mathrm{mg}, 0.086 \mathrm{mmol})$ using the general procedure for $O$-alkylation (GP1 with 1.5 equiv of $\mathrm{NaH}$ and 5 equiv of propargyl bromide; TLC/EtOAc; $\left.R_{f}=0.45\right)$ and the general procedure for deacetylation (GP2; TLC: Tol/ $\mathrm{CH}_{3} \mathrm{OH}$ $\left.(4: 1, \mathrm{v} / \mathrm{v}) ; R_{f}=0.40\right)$, successively. The compound was purified using flash chromatography $\left(\mathrm{CH}_{2} \mathrm{Cl}_{2} / \mathrm{CH}_{3} \mathrm{OH}\right.$, gradient $\left.0-10 \%\right)$ to give 23 $(26.5 \mathrm{mg}, 74 \%)$ as a pale-yellow solid. ${ }^{1} \mathrm{H}$ NMR $\left(\mathrm{CD}_{3} \mathrm{OD}, 600 \mathrm{MHz}\right)$ : $\delta 7.56(\mathrm{dd}, 2 \mathrm{H}, J=8.0,1.4 \mathrm{~Hz}), 7.43(\mathrm{dd}, 1 \mathrm{H}, J=7.8,7.0 \mathrm{~Hz}), 7.37$ $(\mathrm{t}, 2 \mathrm{H}, J=7.5 \mathrm{~Hz}), 4.25(\mathrm{~d}, 2 \mathrm{H}, J=2.4 \mathrm{~Hz}), 3.90(\mathrm{t}, 1 \mathrm{H}, J=10.4 \mathrm{~Hz})$, $3.82-3.75(\mathrm{~m}, 2 \mathrm{H}), 3.70-3.58(\mathrm{~m}, 2 \mathrm{H}), 3.65(\mathrm{~s}, 3 \mathrm{H}), 3.51-3.44(\mathrm{~m}$, $2 \mathrm{H}), 3.09(\mathrm{dd}, 1 \mathrm{H}, J=12.9,4.7 \mathrm{~Hz}), 2.92(\mathrm{t}, 1 \mathrm{H}, J=2.41 \mathrm{~Hz}), 1.96$ $(\mathrm{s}, 3 \mathrm{H}), 1.80(\mathrm{dd}, 1 \mathrm{H}, J=12.9,11.3 \mathrm{~Hz}) .{ }^{13} \mathrm{C}\left\{{ }^{1} \mathrm{H}\right\} \mathrm{NMR}\left(\mathrm{CD}_{3} \mathrm{OD}\right.$, $150 \mathrm{MHz}): \delta 174.7,170.9,137.9,131.2,130.1,129.9,87.9,80.6,77.2$, $76.3,76.2,72.9,70.0,64.5,57.7,53.3,51.7,38.9,22.7$. HRMS (ESITOF) $\mathrm{m} / z:[\mathrm{M}+\mathrm{Na}]^{+}$calcd for $\mathrm{C}_{21} \mathrm{H}_{27} \mathrm{NO}_{8} \mathrm{SNa}$, 476.1350; found, 476.1368.

Methyl (Methyl 5-acetamido-4-O-(tert-butyldimethylsilyl)-3,5dideoxy-8,9-O-isopropylidene-7-propargyloxy-D-glycero- $\alpha$-D-galacto-2-nonulopyranosid)onate (24). Compound $\mathbf{2 4}$ was synthesized starting from 7 ( $40.3 \mathrm{mg}, 0.086 \mathrm{mmol})$ using the general procedure for $O$-alkylation (GP1 with 1.5 equiv of $\mathrm{NaH}$ and 5 equiv of propargyl bromide; TLC: petroleum ether/EtOAc $\left.(2: 3, \mathrm{v} / \mathrm{v}) ; R_{f}=0.61\right)$. The compound was purified using flash chromatography (petroleum ether/EtOAc, gradient $0-60 \%)$ to give $24(22.1 \mathrm{mg}, 57 \%)$ as a paleyellow solid. ${ }^{1} \mathrm{H}$ NMR $\left(\mathrm{CDCl}_{3}, 600 \mathrm{MHz}\right): \delta 5.62(\mathrm{~d}, 1 \mathrm{H}, J=7.5$ $\mathrm{Hz}), 4.54(\mathrm{ABdq}, 2 \mathrm{H}, J=16.2,2.4 \mathrm{~Hz}), 4.36-4.26(\mathrm{~m}, 2 \mathrm{H}), 4.24(\mathrm{dd}$, $1 \mathrm{H}, J=10.8,2.0 \mathrm{~Hz}), 4.16-4.09(\mathrm{~m}, 3 \mathrm{H}), 3.82(\mathrm{~s}, 3 \mathrm{H}), 3.32-3.24$ $(\mathrm{m}, 1 \mathrm{H}), 3.31(\mathrm{~s}, 3 \mathrm{H}), 2.57(\mathrm{dd}, 1 \mathrm{H} J=12.8,4.7 \mathrm{~Hz}), 2.49(\mathrm{t}, 1 \mathrm{H}, J=$ $2.3 \mathrm{~Hz}), 1.93(\mathrm{~s}, 3 \mathrm{H}), 1.68($ appt, $1 \mathrm{H}, J=12.3 \mathrm{~Hz}), 1.43(\mathrm{~s}, 3 \mathrm{H}), 1.35$ $(\mathrm{s}, 3 \mathrm{H}), 0.86(\mathrm{~s}, 9 \mathrm{H}), 0.05(\mathrm{~s}, 3 \mathrm{H}), 0.01(\mathrm{~s}, 3 \mathrm{H}) .{ }^{13} \mathrm{C}\left\{{ }^{1} \mathrm{H}\right\}$ NMR $\left(\mathrm{CDCl}_{3}, 150 \mathrm{MHz}\right): \delta 170.2,168.4,107.9,99.4,81.5,79.1,74.3,74.2$, 72.6, 66.1, 65.1, 59.9, 55.9, 52.6, 52.0, 41.8, 26.5, 25.8, 25.0, 24.0, $18.0,-4.5,-4.8$. HRMS (ESI-TOF) $m / z:[\mathrm{M}+\mathrm{Na}]^{+}$calcd for $\mathrm{C}_{25} \mathrm{H}_{43} \mathrm{NO}_{9} \mathrm{SiNa}$, 552.2599; found, 552.2614.

Methyl (Methyl 5-acetamido-4-O-(tert-butyldimethylsilyl)-3,5dideoxy-8,9-O-isopropylidene-7-propargyloxy-D-glycero- $\alpha$-D-galacto-2-nonulopyranosid)onate (25). Compound 25 was synthesized starting from $8(49.2 \mathrm{mg}, 0.086 \mathrm{mmol})$ using the general procedure for $\mathrm{O}$-alkylation (GP1 with 1.5 equiv of $\mathrm{NaH}$ and 5 equiv of propargyl bromide; TLC: petroleum ether/EtOAc $\left.(3: 2, \mathrm{v} / \mathrm{v}) ; R_{f}=0.24\right)$. The compound was purified using flash chromatography (petroleum ether/EtOAc, gradient $0-50 \%)$ to give $25(37.1 \mathrm{mg}, 71 \%)$ as a paleyellow solid. ${ }^{1} \mathrm{H}$ NMR $\left(\mathrm{CD}_{3} \mathrm{OD}, 600 \mathrm{MHz}\right): \delta 7.55(\mathrm{dd}, 2 \mathrm{H}, J=8.3$, $1.4 \mathrm{~Hz}), 7.44(\mathrm{t}, 1 \mathrm{H}, J=7.4 \mathrm{~Hz}), 7.38(\mathrm{t}, 2 \mathrm{H}, J=7.5 \mathrm{~Hz}), 4.40$ (ABdq, $2 \mathrm{H}, J=15.0,2.5 \mathrm{~Hz}), 4.11(\mathrm{ddd}, 1 \mathrm{H}, J=7.6,6.4,4.2 \mathrm{~Hz})$, $4.03(\mathrm{dd}, 1 \mathrm{H}, J=8.5,7.7 \mathrm{~Hz}, 1 \mathrm{H}), 3.89$ (dd, $1 \mathrm{H}, J=8.5,6.5 \mathrm{~Hz}), 3.80$ $(\mathrm{t}, 1 \mathrm{H}, J=10.7 \mathrm{~Hz}), 3.77(\mathrm{~d}, 1 \mathrm{H}, J=4.1 \mathrm{~Hz}), 3.59(\mathrm{~s}, 3 \mathrm{H}), 3.66-3.57$ $(\mathrm{m}, 1 \mathrm{H}), 3.48(\mathrm{~d}, 1 \mathrm{H}, J=10.6 \mathrm{~Hz}), 2.90(\mathrm{t}, 1 \mathrm{H}, J=2.4 \mathrm{~Hz}), 2.67(\mathrm{dd}$, $1 \mathrm{H}, J=12.9,4.6 \mathrm{~Hz}), 1.93(\mathrm{~s}, 3 \mathrm{H}), 1.74(\mathrm{dd}, 1 \mathrm{H}, J=12.9,11.2 \mathrm{~Hz})$, $1.38(\mathrm{~s}, 3 \mathrm{H}), 1.25(\mathrm{~s}, 3 \mathrm{H}), 0.87(\mathrm{~s}, 9 \mathrm{H}), 0.07(\mathrm{~s}, 3 \mathrm{H}), 0.04(\mathrm{~s}, 3 \mathrm{H})$. 
${ }^{13} \mathrm{C}\left\{{ }^{1} \mathrm{H}\right\}$ NMR $\left(\mathrm{CD}_{3} \mathrm{OD}, 150 \mathrm{MHz}\right): \delta 173.0,170.5,138.0,131.1$, $130.5,129.9,109.3,88.6,81.0,78.8,77.3,76.6,76.2,71.3,67.1,61.1$, 53.3, 52.9, 42.7, 26.8, 26.1, 25.6, 23.3, 18.7, -4.4, -4.7. HRMS (ESITOF) $m / z:[\mathrm{M}+\mathrm{Na}]^{+}$calcd for $\mathrm{C}_{30} \mathrm{H}_{45} \mathrm{NO}_{8} \mathrm{SSiNa}, 630.2527$; found, 630.2553.

Methyl (Methyl 5-acetamido-3,5-dideoxy-8,9-O-isopropylideneD-glycero- $\alpha$-D-galacto-2-nonulopyranosid)onate (26). ${ }^{\prime}$ Compound 26 was synthesized in $88 \%$ yield following the procedure described in ref $1 .{ }^{1} \mathrm{H}$ NMR ( $\left.\mathrm{CD}_{3} \mathrm{OD}, 600 \mathrm{MHz}\right): \delta 4.25(\mathrm{q}, 1 \mathrm{H}, J=6.3 \mathrm{~Hz}), 4.08$ $(\mathrm{dd}, 1 \mathrm{H}, J=8.3,6.4 \mathrm{~Hz}), 4.00(\mathrm{dd}, 1 \mathrm{H}, J=8.3,6.5 \mathrm{~Hz}), 3.80(\mathrm{~s}, 3 \mathrm{H})$, $3.77(\mathrm{~d}, 1 \mathrm{H}, J=10.3 \mathrm{~Hz}$ ), $3.63(\mathrm{ddd}, 1 \mathrm{H}, J=12.0,10.1,4.7 \mathrm{~Hz}), 3.60$ $(\mathrm{d}, 1 \mathrm{H}, J=6.2 \mathrm{~Hz}), 3.55$ (dd, $1 \mathrm{H}, J=10.5,1.5 \mathrm{~Hz}), 3.35(\mathrm{~s}, 3 \mathrm{H}), 2.61$ $(\mathrm{dd}, 1 \mathrm{H}, J=12.8,4.6 \mathrm{~Hz}), 1.99(\mathrm{~s}, 3 \mathrm{H}), 1.67(\mathrm{dd}, 1 \mathrm{H}, J=12.7,11.9$ $\mathrm{Hz}), 1.37(\mathrm{~s}, 3 \mathrm{H}), 1.34(\mathrm{~s}, 3 \mathrm{H}) .{ }^{13} \mathrm{C}\left\{{ }^{1} \mathrm{H}\right\} \mathrm{NMR}\left(\mathrm{CD}_{3} \mathrm{OD}, 150 \mathrm{MHz}\right)$ : $\delta 174.8,170.1,109.8,100.6,77.4,75.4,70.6,68.6,67.4,53.8,52.8$, 52.1, 41.4, 27.1, 25.8, 22.7. HRMS (ESI-TOF) $\mathrm{m} / z:[\mathrm{M}+\mathrm{Na}]^{+}$calcd for $\mathrm{C}_{16} \mathrm{H}_{27} \mathrm{NO}_{9} \mathrm{Na}$, 400.1578; found, 400.1596 .

Methyl (Phenyl 5-acetamido-3,5-dideoxy-8,9-0-isopropylidene2-thio-D-glycero- $\alpha$-D-galacto-2-nonulopyranosid)onate (27). ${ }^{4}$ Compound 27 was synthesized in $92 \%$ yield following the procedure described in ref $4 .{ }^{1} \mathrm{H}$ NMR $\left(\mathrm{CD}_{3} \mathrm{OD}, 600 \mathrm{MHz}\right): \delta 7.58(\mathrm{dd}, 2 \mathrm{H}, J=$ $7.6,1.6 \mathrm{~Hz}), 7.42(\mathrm{t}, 1 \mathrm{H}, J=7.2 \mathrm{~Hz}), 7.36(\mathrm{t}, 2 \mathrm{H}, J=7.6 \mathrm{~Hz}), 4.17(\mathrm{q}$, $1 \mathrm{H}, J=6.7 \mathrm{~Hz}$ ), $3.97(\mathrm{dd}, 1 \mathrm{H}, J=8.3,6.2 \mathrm{~Hz}, 1 \mathrm{H}), 3.88(\mathrm{dd}, 1 \mathrm{H}, J=$ 8.4, $6.7 \mathrm{~Hz}), 3.79(\mathrm{t}, 1 \mathrm{H}, J=10.3 \mathrm{~Hz}), 3.61(\mathrm{ddd}, 1 \mathrm{H}, J=11.3,10.1$, $4.7 \mathrm{~Hz}), 3.51-3.47(\mathrm{~m}, 1 \mathrm{H}), 3.48(\mathrm{~s}, 3 \mathrm{H}), 3.24(\mathrm{~d}, 1 \mathrm{H}, J=10.7 \mathrm{~Hz})$, $2.81(\mathrm{dd}, 1 \mathrm{H}, J=12.7,4.8 \mathrm{~Hz}), 1.97(\mathrm{~s}, 3 \mathrm{H}), 1.77(\mathrm{dd}, 1 \mathrm{H}, J=12.8$, $11.3 \mathrm{~Hz}), 1.31(\mathrm{~s}, 3 \mathrm{H}), 1.22(\mathrm{~s}, 3 \mathrm{H}) .{ }^{13} \mathrm{C}\left\{{ }^{1} \mathrm{H}\right\}$ NMR ( $\mathrm{CD}_{3} \mathrm{OD}, 150$ $\mathrm{MHz}): \delta 174.8,170.5,137.9,130.9,130.8,129.7,109.8,88.6,77.6$, 76.7, 71.2., 69.0, 68.0, 53.5, 52.6, 42.0, 27.0, 25.7, 22.7. HRMS (ESITOF) $m / z:[\mathrm{M}+\mathrm{Na}]^{+}$calcd for $\mathrm{C}_{21} \mathrm{H}_{29} \mathrm{NO}_{8} \mathrm{SNa}$, 478.1506; found, 478.1523.

Methyl (Methyl 5-acetamido-3,5-dideoxy-8,9-O-isopropylidene4-propargyloxy-D-glycero- $\alpha$-D-galacto-2-nonulopyranosid)onate (28). Compound 28 was synthesized starting from 26 (32.6 mg, 0.086 $\mathrm{mmol}$ ) using the general procedure for $\mathrm{O}$-alkylation (GP1 with 1.1 equiv of $\mathrm{NaH}$ and 5 equiv of propargyl bromide; TLC: petroleum ether/EtOAc $\left.(1: 4, \mathrm{v} / \mathrm{v}) ; R_{f}=0.21\right)$. The compound was purified using flash chromatography (petroleum ether/EtOAc, gradient 30$90 \%)$ to give $28(11 \mathrm{mg}, 31 \%)$ as a white solid. ${ }^{1} \mathrm{H}$ NMR $\left(\mathrm{CD}_{3} \mathrm{OD}\right.$, $600 \mathrm{MHz}): \delta 4.24(\mathrm{q}, 1 \mathrm{H}, J=6.3 \mathrm{~Hz}), 4.21(\mathrm{t}, 2 \mathrm{H}, J=2.2 \mathrm{~Hz}), 4.04$ $(\mathrm{ABdq}, 2 \mathrm{H}, J=8.3,6.3 \mathrm{~Hz}), 3.88(\mathrm{t}, 1 \mathrm{H}, J=10.3 \mathrm{~Hz}), 3.81(\mathrm{~s}, 3 \mathrm{H})$, 3.69 (ddd, $1 \mathrm{H}, J=11.4,10.0,4.5 \mathrm{~Hz}), 3.65(\mathrm{~d}, 1 \mathrm{H}, J=10.7 \mathrm{~Hz}), 3.60$ $(\mathrm{d}, 1 \mathrm{H}, J=6.3 \mathrm{~Hz}), 3.35(\mathrm{~s}, 3 \mathrm{H}), 2.88(\mathrm{t}, 1 \mathrm{H}, J=2.4 \mathrm{~Hz}), 2.81(\mathrm{dd}$, $1 \mathrm{H}, J=12.7,4.7 \mathrm{~Hz}), 1.97(\mathrm{~s}, 3 \mathrm{H}), 1.61(\mathrm{dd}, 1 \mathrm{H}, J=12.8,11.6 \mathrm{~Hz})$, $1.37(\mathrm{~s}, 3 \mathrm{H}), 1.34(\mathrm{~s}, 3 \mathrm{H}) .{ }^{13} \mathrm{C}\left\{{ }^{1} \mathrm{H}\right\} \mathrm{NMR}\left(\mathrm{CD}_{3} \mathrm{OD}, 150 \mathrm{MHz}\right): \delta$ $174.31,170.0,109.8,100.6,80.7,77.4,76.0,75.8,75.1,70.4,67.4$, 57.5, 52.8, 52.1, 51.9, 38.4, 27.1, 25.8, 22.8. HRMS (ESI-TOF) $\mathrm{m} / \mathrm{z}$ : $[\mathrm{M}+\mathrm{Na}]^{+}$calcd for $\mathrm{C}_{19} \mathrm{H}_{29} \mathrm{NO}_{9} \mathrm{Na}$, 438.1735; found, 438.1749.

Methyl (Phenyl 5-acetamido-3,5-dideoxy-8,9-O-isopropylidene4-propargyloxy-2-thio-D-glycero- $\alpha$-D-galacto-2-nonulopyranosid)onate (29). Compound 29 was synthesized starting from 27 (39.3 $\mathrm{mg}, 0.086 \mathrm{mmol}$ ) using the general procedure for $O$-alkylation (GP1 with 1.1 equiv of $\mathrm{NaH}$ and 5 equiv of propargyl bromide; TLC: petroleum ether/EtOAc $\left.(1: 4, \mathrm{v} / \mathrm{v}) ; R_{f}=0.29\right)$. The compound was purified using flash chromatography (petroleum ether/EtOAc, gradient $20-90 \%)$ to give $29(20.9 \mathrm{mg}, 49 \%)$ as a pale-yellow solid. ${ }^{1} \mathrm{H}$ NMR ( $\left.\mathrm{CD}_{3} \mathrm{OD}, 600 \mathrm{MHz}\right): \delta 7.58(\mathrm{dd}, 2 \mathrm{H}, J=8.3,1.6$ $\mathrm{Hz}), 7.42(\mathrm{dd}, 1 \mathrm{H}, J=7.7,6.9 \mathrm{~Hz}), 7.36(\mathrm{t}, 2 \mathrm{H}, J=7.6 \mathrm{~Hz}), 4.26-$ $4.18(\mathrm{~m}, 2 \mathrm{H}), 4.15(\mathrm{q}, 1 \mathrm{H}, J=6.7 \mathrm{~Hz}, 1 \mathrm{H}), 3.97(\mathrm{dd}, 1 \mathrm{H}, J=8.4,6.3$ $\mathrm{Hz}), 3.91-3.85(\mathrm{~m}, 2 \mathrm{H}), 3.65(\mathrm{dt}, 1 \mathrm{H}, J=10.7,4.9 \mathrm{~Hz}), 3.50(\mathrm{~s}, 3 \mathrm{H})$, $3.52-3.48(\mathrm{~m}, 1 \mathrm{H}), 3.45(\mathrm{dd}, 1 \mathrm{H}, J=10.9,1.5 \mathrm{~Hz}), 3.02(\mathrm{dd}, 1 \mathrm{H}, J=$ $12.7,4.9 \mathrm{~Hz}), 2.87(\mathrm{t}, 1 \mathrm{H}, J=2.5 \mathrm{~Hz}), 1.95(\mathrm{~s}, 3 \mathrm{H}), 1.70(\mathrm{dd}, 1 \mathrm{H}, J=$ 12.7, $11.2 \mathrm{~Hz}), 1.31(\mathrm{~s}, 3 \mathrm{H}), 1.22(\mathrm{~s}, 3 \mathrm{H}) .{ }^{13} \mathrm{C}\left\{{ }^{1} \mathrm{H}\right\} \mathrm{NMR}\left(\mathrm{CD}_{3} \mathrm{OD}\right.$, $150 \mathrm{MHz}): \delta 174.3,170.3,137.9,131.0,130.7,129.7,109.9,88.5$, 80.7, 77.4, 76.7, 76.4, 76.1, 71.0, 68.0, 57.7, 52.6, 51.6, 39.1, 27.0, 25.7, 22.8. HRMS (ESI-TOF) $\mathrm{m} / z:[\mathrm{M}+\mathrm{Na}]^{+}$calcd for $\mathrm{C}_{24} \mathrm{H}_{31} \mathrm{NO}_{8} \mathrm{SNa}$, 516.1663; found, 516.1683.

Methyl (Methyl 5-acetamido-4-benzyloxy-3,5-dideoxy-D-glycero- $\alpha$-D-galacto-2-nonulopyranosid)onate (30). Compound 30 was synthesized starting from $6(40 \mathrm{mg}, 0.086 \mathrm{mmol})$ using the general procedure for $\mathrm{O}$-alkylation (GP1 with 1.1 equiv of $\mathrm{NaH}$ and 5 equiv of benzyl bromide; TLC/EtOAc; $\left.R_{f}=0.57\right)$ and the general procedure for deacetylation (GP2), successively. The compound was purified using flash chromatography $\left(\mathrm{Tol} / \mathrm{CH}_{3} \mathrm{OH}\right.$, gradient 5-20\%) to give $30(22.6 \mathrm{mg}, 64 \%)$ as a white solid. ${ }^{1} \mathrm{H}$ NMR $\left(\mathrm{CD}_{3} \mathrm{OD}, 600\right.$ $\mathrm{MHz}): \delta 7.47-7.21(\mathrm{~m}, 5 \mathrm{H}), 4.58(\mathrm{ABq}, 2 \mathrm{H}, J=11.9 \mathrm{~Hz}), 3.94(\mathrm{t}$, $1 \mathrm{H}, J=10.3 \mathrm{~Hz}), 3.81(\mathrm{~s}, 3 \mathrm{H}), 3.87-3.79(\mathrm{~m}, 2 \mathrm{H}), 3.68-3.62(\mathrm{~m}$, $2 \mathrm{H}), 3.57(\mathrm{ddd}, 1 \mathrm{H}, J=11.7,10.1,4.6 \mathrm{~Hz}), 3.52(\mathrm{dd}, 1 \mathrm{H}, J=9.0,1.7$ $\mathrm{Hz}), 3.34(\mathrm{~s}, 3 \mathrm{H}), 2.78(\mathrm{dd}, 1 \mathrm{H}, J=12.8,4.6 \mathrm{~Hz}), 1.96(\mathrm{~s}, 3 \mathrm{H}), 1.68$ $($ dd, $1 \mathrm{H}, J=12.8,11.7 \mathrm{~Hz}) \cdot{ }^{13} \mathrm{C}\left\{{ }^{1} \mathrm{H}\right\}$ NMR $\left(\mathrm{CD}_{3} \mathrm{OD}, 150 \mathrm{MHz}\right): \delta$ 174.6, 170.7, 139.6, 129.4, 128.9, 128.8, 100.4, 76.1, 74.6, 72.4, 72.2, 70.1, 64.7, 53.3, 52.0, 38.6, 22.7. HRMS (ESI-TOF) $m / z:[\mathrm{M}+\mathrm{Na}]^{+}$ calcd for $\mathrm{C}_{20} \mathrm{H}_{29} \mathrm{NO}_{9} \mathrm{Na}$, 450.1735; found, 450.1749.

Methyl (Methyl 5-acetamido-4-allyloxy-3,5-dideoxy-D-glycero- $\alpha$ D-galacto-2-nonulopyranosid)onate (31). Compound 31 was synthesized starting from $6(40 \mathrm{mg}, 0.086 \mathrm{mmol})$ using the general procedure for $O$-alkylation (GP1 with 1.1 equiv of $\mathrm{NaH}$ and 5 equiv of allyl bromide; TLC/EtOAc; $\left.R_{f}=0.43\right)$ and the general procedure for deacetylation (GP2), successively. The compound was purified using flash chromatography $\left(\mathrm{Tol} / \mathrm{CH}_{3} \mathrm{OH}\right.$, gradient 5-20\%) to give $31(17 \mathrm{mg}, 52 \%)$ as a white solid. ${ }^{1} \mathrm{H}$ NMR $\left(\mathrm{CD}_{3} \mathrm{OD}, 600 \mathrm{MHz}\right): \delta$ $5.89(\mathrm{ddt}, 1 \mathrm{H}, J=17.3,10.4,5.5 \mathrm{~Hz}), 5.27(\mathrm{qd}, 1 \mathrm{H}, J=17.2,1.8 \mathrm{~Hz})$, $5.16(\mathrm{qd}, 1 \mathrm{H}, J=10.5,1.4 \mathrm{~Hz}), 4.12(\mathrm{ddt}, 1 \mathrm{H}, J=12.9,5.5,1.6 \mathrm{~Hz})$, 3.99 (ddd, $1 \mathrm{H}, J=12.8,5.7,1.6 \mathrm{~Hz}), 3.91-3.80(\mathrm{~m}, 3 \mathrm{H}), 3.84(\mathrm{~s}$, $3 \mathrm{H}), 3.68-3.60(\mathrm{~m}, 2 \mathrm{H}), 3.56-3.48(\mathrm{~m}, 2 \mathrm{H}), 3.35(\mathrm{~s}, 3 \mathrm{H}), 2.79(\mathrm{dd}$, $1 \mathrm{H}, J=12.8,4.6 \mathrm{~Hz}), 1.98(\mathrm{~s}, 3 \mathrm{H}), 1.64(\mathrm{dd}, 1 \mathrm{H}, J=12.8,11.7 \mathrm{~Hz}$, 1H). ${ }^{13} \mathrm{C}\left\{{ }^{1} \mathrm{H}\right\}$ NMR $\left(\mathrm{CD}_{3} \mathrm{OD}, 150 \mathrm{MHz}\right): \delta 174.7,170.7,136.1$, 117.2, 100.4, 75.9, 74.7, 72.4, 71.2, 70.2, 64.7, 53.3, 52.1, 52.0, 38.6, 22.7. HRMS (ESI-TOF) $m / z$ : $[\mathrm{M}+\mathrm{Na}]^{+}$calcd for $\mathrm{C}_{16} \mathrm{H}_{27} \mathrm{NO}_{9} \mathrm{Na}$, 400.1578; found, 400.1587 .

Methyl (Methyl 5-acetamido-3,5-dideoxy-4-(2-methoxy-2-oxoethoxy)- $D$-glycero- $\alpha$-D-galacto-2-nonulopyranosid)onate (32). Compound 32 was synthesized starting from $6(40 \mathrm{mg}, 0.086 \mathrm{mmol})$ using the general procedure for $O$-alkylation (GP1 with 1.1 equiv of $\mathrm{NaH}$ and 5 equiv of methyl bromoacetate; TLC/EtOAc; $\left.R_{f}=0.35\right)$ and the general procedure for deacetylation (GP2), successively. The compound was purified using flash chromatography $\left(\mathrm{Tol} / \mathrm{CH}_{3} \mathrm{OH}\right.$, gradient $5-20 \%)$ to give $32(28 \mathrm{mg}, 79 \%)$ as a pale-yellow solid. ${ }^{1} \mathrm{H}$ NMR ( $\left.\mathrm{CD}_{3} \mathrm{OD}, 600 \mathrm{MHz}\right): \delta 4.21(\mathrm{ABq}, 2 \mathrm{H}, J=16.8 \mathrm{~Hz}), 3.89-$ $3.81(\mathrm{~m}, 3 \mathrm{H}), 3.84(\mathrm{~s}, 3 \mathrm{H}), 3.75(\mathrm{~s}, 3 \mathrm{H}), 3.67-3.56(\mathrm{~m}, 3 \mathrm{H}), 3.52$ $(\mathrm{dd}, 1 \mathrm{H}, J=8.9,1.6 \mathrm{~Hz}), 3.35(\mathrm{~s}, 3 \mathrm{H}), 2.82(\mathrm{dd}, 1 \mathrm{H}, J=12.9,4.7$ $\mathrm{Hz}), 2.01(\mathrm{~s}, 3 \mathrm{H}), 1.69(\mathrm{dd}, 1 \mathrm{H}, J=12.9,11.7 \mathrm{~Hz}) .{ }^{13} \mathrm{C}\left\{{ }^{1} \mathrm{H}\right\} \mathrm{NMR}$ $\left(\mathrm{CD}_{3} \mathrm{OD}, 150 \mathrm{MHz}\right): \delta 175.1,173.0,170.7,100.3,77.3,74.8,72.3$, $70.2,67.2,64.7,53.4,52.4,52.3,52.0,38.3,22.8$. HRMS (ESI-TOF) $m / z:[\mathrm{M}+\mathrm{Na}]^{+}$calcd for $\mathrm{C}_{16} \mathrm{H}_{27} \mathrm{NO}_{11} \mathrm{Na}, 432.1476$; found, 432.1491 .

Methyl (Methyl 5-acetamido-3,5-dideoxy-4-(hex-5-yn-1-yloxy)$D$-glycero- $\alpha$-D-galacto-2-nonulopyranosid)onate (33). Compound 33 was synthesized starting from $6(40 \mathrm{mg}, 0.086 \mathrm{mmol})$ using the general procedure for $O$-alkylation (GP1 with 3 equiv of $\mathrm{NaH}, 5$ equiv of 6-iodo-1-hexyne and DMF as solvent) and the general procedure for deacetylation (GP2; TLC: $\left.\mathrm{Tol} / \mathrm{CH}_{3} \mathrm{OH}(4: 1, \mathrm{v} / \mathrm{v}) ; R_{f}=0.37\right)$, successively. The compound was purified using flash chromatography $\left(\mathrm{Tol} / \mathrm{CH}_{3} \mathrm{OH}\right.$, gradient $\left.5-20 \%\right)$ to give $33(14.4 \mathrm{mg}, 40 \%)$ as a paleyellow solid. ${ }^{1} \mathrm{H}$ NMR $\left(\mathrm{CD}_{3} \mathrm{OD}, 600 \mathrm{MHz}\right): \delta 3.90-3.81(\mathrm{~m}, 3 \mathrm{H})$, $3.85(\mathrm{~s}, 3 \mathrm{H}), 3.68-3.60(\mathrm{~m}, 3 \mathrm{H}), 3.51(\mathrm{dd}, 1 \mathrm{H}, J=9.0,1.7 \mathrm{~Hz})$, $3.48-3.38(\mathrm{~m}, 2 \mathrm{H}), 3.03(\mathrm{~s}, 3 \mathrm{H}), 2.78(\mathrm{dd}, 1 \mathrm{H}, J=12.8,4.6 \mathrm{~Hz})$, $2.23-2.15(\mathrm{~m}, 3 \mathrm{H}), 1.99(\mathrm{~s}, 3 \mathrm{H}), 1.70-1.53(\mathrm{~m}, 5 \mathrm{H}) .{ }^{13} \mathrm{C}\left\{{ }^{1} \mathrm{H}\right\} \mathrm{NMR}$ $\left(\mathrm{CD}_{3} \mathrm{OD}, 150 \mathrm{MHz}\right): \delta 174.7,170.8,100.4,84.9,76.5,74.7,72.4$, 70.2, 69.7, 64.8, 53.3, 52.1, 52.0, 38.5, 30.1, 26.5, 22.7, 18.8. HRMS (ESI-TOF) $\mathrm{m} / z$ : $[\mathrm{M}+\mathrm{Na}]^{+}$calcd for $\mathrm{C}_{19} \mathrm{H}_{31} \mathrm{NO}_{9} \mathrm{Na}$, 440.1891; found, 440.1904 .

Methyl (Methyl 5-acetamido-3,5-dideoxy-4-(pent-4-en-1-yloxy)$D$-glycero- $\alpha$-D-galacto-2-nonulopyranosid)onate (34). Compound 34 was synthesized starting from $6(40 \mathrm{mg}, 0.086 \mathrm{mmol})$ using the general procedure for $\mathrm{O}$-alkylation (GP1 with 2 equiv of $\mathrm{NaH}, 5$ equiv of 5-bromo-1-pentene and DMF as solvent) and the general procedure for deacetylation (GP2; TLC: Tol/ $\mathrm{CH}_{3} \mathrm{OH}(4: 1, \mathrm{v} / \mathrm{v})$; $R_{f}=0.33$ ). The compound was purified using flash chromatography $\left(\mathrm{Tol} / \mathrm{CH}_{3} \mathrm{OH}\right.$, gradient $\left.5-20 \%\right)$ to give $34(6 \mathrm{mg}, 17 \%)$ as a pale- 
yellow solid. ${ }^{1} \mathrm{H}$ NMR $\left(\mathrm{CD}_{3} \mathrm{OD}, 600 \mathrm{MHz}\right): \delta 5.84(\mathrm{ddt}, 1 \mathrm{H}, J=$ $17.1,10.3,6.7 \mathrm{~Hz}), 5.03(\mathrm{qd}, 1 \mathrm{H}, J=17.1,1.8 \mathrm{~Hz}), 4.97(\mathrm{dd}, 1 \mathrm{H}, J=$ $10.2,2.1 \mathrm{~Hz}), 3.90-3.83(\mathrm{~m}, 3 \mathrm{H}), 3.87(\mathrm{~s}, 3 \mathrm{H}), 3.69-3.61(\mathrm{~m}, 3 \mathrm{H})$, $3.53(\mathrm{dd}, 1 \mathrm{H}, J=8.9,1.8 \mathrm{~Hz}), 3.49-3.40(\mathrm{~m}, 2 \mathrm{H}), 3.37(\mathrm{~s}, 3 \mathrm{H}), 2.78$ $(\mathrm{dd}, 1 \mathrm{H}, J=12.8,4.6 \mathrm{~Hz}), 2.16-2.10(\mathrm{~m}, 2 \mathrm{H}), 1.99(\mathrm{~s}, 3 \mathrm{H}), 1.69-$ $1.60(\mathrm{~m}, 3 \mathrm{H}) .{ }^{13} \mathrm{C}\left\{{ }^{1} \mathrm{H}\right\}$ NMR $\left(\mathrm{CD}_{3} \mathrm{OD}, 150 \mathrm{MHz}\right): \delta 174.6,170.8$, 139.4, 115.3, 100.4, 76.5, 74.7, 72.4, 70.2, 69.6, 64.8, 53.3, 52.1, 52.0, $38.5,31.3,30.4,22.7$. HRMS (ESI-TOF) $m / z:[\mathrm{M}+\mathrm{Na}]^{+}$calcd for $\mathrm{C}_{18} \mathrm{H}_{31} \mathrm{NO}_{9} \mathrm{Na}, 428.1891$; found, 428.1904 .

Methyl (Methyl 5-acetamido-3,5-dideoxy-4-ethoxy-D-glycero- $\alpha$ D-galacto-2-nonulopyranosid)onate (35). Compound 35 was synthesized starting from $6(40 \mathrm{mg}, 0.086 \mathrm{mmol})$ using the general procedure for $O$-alkylation (GP1 with 1.1 equiv of $\mathrm{NaH}$ and 5 equiv of ethyl tosylate; TLC/EtOAc; $\left.R_{f}=0.38\right)$ and the general procedure for deacetylation (GP2). The compound was purified using flash chromatography $\left(\mathrm{Tol} / \mathrm{CH}_{3} \mathrm{OH}\right.$, gradient $\left.5-30 \%\right)$ to give $35(2.5 \mathrm{mg}$, $8 \%)$ as a pale-yellow oil. After flash chromatography and reversephase HPLC purification $\left(\mathrm{H}_{2} \mathrm{O} /\right.$ acetonitrile w. $0.005 \%$ formic acid, gradient $5-65 \%$ over $25 \mathrm{~min}$ ), the product still contained $20 \%$ of tosyl-containing byproducts. ${ }^{1} \mathrm{H}$ NMR $\left(\mathrm{CD}_{3} \mathrm{OD}, 600 \mathrm{MHz}\right): \delta 3.88-$ $3.79(\mathrm{~m}, 3 \mathrm{H}), 3.85(\mathrm{~s}, 3 \mathrm{H}), 3.69-3.62(\mathrm{~m}, 2 \mathrm{H}), 3.60(\mathrm{dd}, 1 \mathrm{H}, J=$ $10.8,1.7 \mathrm{~Hz}), 3.53-3.43(\mathrm{~m}, 3 \mathrm{H}), 3.35(\mathrm{~s}, 3 \mathrm{H}), 2.77(\mathrm{dd}, 1 \mathrm{H}, J=$ 12.8, $4.6 \mathrm{~Hz}), 1.98(\mathrm{~s}, 3 \mathrm{H}), 1.62(\mathrm{dd}, 1 \mathrm{H}, J=12.9,11.7 \mathrm{~Hz}), 1.16(\mathrm{t}$, $3 \mathrm{H}, J=7.1 \mathrm{~Hz}) \cdot{ }^{13} \mathrm{C}\left\{{ }^{1} \mathrm{H}\right\} \mathrm{NMR}\left(\mathrm{CD}_{3} \mathrm{OD}, 150 \mathrm{MHz}\right): \delta 174.8,170.8$, 100.4, 76.2, 74.8, 72.4, 70.2, 65.7, 64.7, 53.3, 52.2, 52.0, 38.6, 22.6, 15.9. HRMS (ESI-TOF) $m / z$ : $[\mathrm{M}+\mathrm{Na}]^{+}$calcd for $\mathrm{C}_{15} \mathrm{H}_{27} \mathrm{NO}_{9} \mathrm{Na}$, 388.1578; found, 388.1594 .

Methyl (Phenyl 5-acetamido-7,8,9-tri-O-acetyl-3,5-dideoxy-4propargyloxy-2-thio-D-glycero- $\alpha$-D-galacto-2-nonulopyranosid)onate (37). Compound 37 was isolated during the synthesis of 23. The compound was purified using flash chromatography ( $n$-hept/ EtOAc, gradient). ${ }^{1} \mathrm{H}$ NMR $\left(\mathrm{CDCl}_{3}, 400 \mathrm{MHz}\right): \delta 7.52-7.47(\mathrm{~m}$, $2 \mathrm{H}), 7.40-7.28(\mathrm{~m}, 3 \mathrm{H}), 5.43(\mathrm{~d}, 1 \mathrm{H}, J=8.8 \mathrm{~Hz}), 5.30-5.22(\mathrm{~m}$, $2 \mathrm{H}), 4.38(\mathrm{dd}, 1 \mathrm{H}, J=12.5,2.1 \mathrm{~Hz}), 4.23(\mathrm{dd}, 1 \mathrm{H}, J=12.3,4.8 \mathrm{~Hz})$, $4.21(\mathrm{dd}, 1 \mathrm{H}, J=16.2,2.2 \mathrm{~Hz}), 4.15-4.04(\mathrm{~m}, 2 \mathrm{H}), 3.86(\mathrm{td}, 1 \mathrm{H}, J=$ $10.8,4.2 \mathrm{~Hz}), 3.54(\mathrm{~s}, 3 \mathrm{H}), 3.39(\operatorname{appq}, 1 \mathrm{H}, J=9.7 \mathrm{~Hz}), 2.97(\mathrm{dd}, 1 \mathrm{H}$, $J=12.9,4.6 \mathrm{~Hz}), 2.42(\mathrm{t}, 1 \mathrm{H}, J=2.4 \mathrm{~Hz}), 2.13(\mathrm{~s}, 3 \mathrm{H}), 2.04(\mathrm{~s}, 3 \mathrm{H})$, $2.02(\mathrm{~s}, 3 \mathrm{H}), 1.93(\mathrm{~s}, 3 \mathrm{H}), 1.74(\mathrm{dd}, 1 \mathrm{H}, J=12.9,11.2 \mathrm{~Hz}) .{ }^{13} \mathrm{C}\left\{{ }^{1} \mathrm{H}\right\}$ $\operatorname{NMR}\left(\mathrm{CDCl}_{3}, 100 \mathrm{MHz}\right): \delta 171.3,170.7,170.7,170.6,170.1,168.2$, 136.5, 129.9, 129.0, 128.9, 87.7, 79.7, 74.8, 73.8, 70.0, 68.3, 62.2, 56.7, $52.7,51.3,38.4,23.8,21.1,21.0,20.9$. HRMS (ESI-TOF) $\mathrm{m} / z$ : $[\mathrm{M}+$ $\mathrm{Na}]^{+}$calcd for $\mathrm{C}_{27} \mathrm{H}_{33} \mathrm{NO}_{11} \mathrm{SNa}, 602.1666$; found, 602.1667.

Methyl 5-Acetamido-7,8,9-tri-O-acetyl-2,6-anhydro-3,5-dideoxy4-propargyloxy-D-glycero- $\alpha$-D-galacto-non-2-enonate (38). Compound $37(19 \mathrm{mg}, 0.033 \mathrm{mmol}$ ) was dissolved in dry dichloromethane $(630 \mu \mathrm{L})$ in the presence of molecular sieves $(3 \AA, 90 \mathrm{mg})$. The mixture was stirred at room temperature and under a nitrogen atmosphere for $16 \mathrm{~h}$. At room temperature, $\mathrm{N}$-iodosuccinimide (14.8 $\mathrm{mg}, 0.066 \mathrm{mmol}, 2$ equiv) and triflic acid $(1 \mu \mathrm{L}, 0.007 \mathrm{mmol}, 0.2$ equiv) were added, and the reaction was allowed to perform for 30 min. After completion, the mixture was diluted with dichloromethane $(5 \mathrm{~mL})$ and washed with a $20 \%$ solution of sodium thiosulfate $(1 \mathrm{~mL})$ and brine $(1 \mathrm{~mL})$ twice, successively. The organic layer was dried over anhydrous sodium sulfate, filtered, and concentrated to dryness. The compound was purified using flash chromatography ( $n$-hept/acetone, gradient $10-50 \%)$ to give $37(13.4 \mathrm{mg}, 87 \%) .{ }^{1} \mathrm{H} \mathrm{NMR}\left(\mathrm{CDCl}_{3}, 400\right.$ $\mathrm{MHz}): \delta 6.16(\mathrm{~d}, 1 \mathrm{H}, J=3.7 \mathrm{~Hz}), 5.63(\mathrm{~d}, 1 \mathrm{H}, J=8.4 \mathrm{~Hz}), 5.53(\mathrm{dd}$, $1 \mathrm{H}, J=5.1,4.8 \mathrm{~Hz}), 5.40(\mathrm{dt}, 1 \mathrm{H}, J=8.1,3.5 \mathrm{~Hz}), 4.50(\mathrm{dd}, 1 \mathrm{H}, J=$ $12.1,3.6 \mathrm{~Hz}), 4.48(\mathrm{dd}, 1 \mathrm{H}, J=7.2,4.7 \mathrm{~Hz}), 4.37(\mathrm{dd}, 1 \mathrm{H}, J=5.3,3.8$ $\mathrm{Hz}), 4.33(\mathrm{dd}, 1 \mathrm{H}, J=16.1,2.3 \mathrm{~Hz}), 4.26(\mathrm{dd}, 1 \mathrm{H}, J=16.1,2.4 \mathrm{~Hz})$, $4.22-4.13(\mathrm{~m}, 2 \mathrm{H}), 3.80(\mathrm{~s}, 3 \mathrm{H}), 2.46(\mathrm{t}, 1 \mathrm{H}, J=2.3 \mathrm{~Hz}), 2.11(\mathrm{~s}$, $3 \mathrm{H}), 2.04(\mathrm{~s}, 3 \mathrm{H}), 2.04(\mathrm{~s}, 3 \mathrm{H}), 1.98(\mathrm{~s}, 3 \mathrm{H}) .{ }^{13} \mathrm{C}\left\{{ }^{1} \mathrm{H}\right\} \mathrm{NMR}\left(\mathrm{CDCl}_{3}\right.$, $100 \mathrm{MHz}): \delta 170.7,170.2,170.2,169.9,162.1,144.1,108.5,79.4$, 76.0, 75.3, 70.6, 69.9, 68.3, 62.0, 56.5, 52.7, 47.8, 23.5, 21.0, 20.9, 20.8. HRMS (ESI-TOF) $m / z:[\mathrm{M}+\mathrm{Na}]^{+}$calcd for $\mathrm{C}_{21} \mathrm{H}_{27} \mathrm{NO}_{11} \mathrm{Na}$, 492.1476; found, 492.1472.

\section{ASSOCIATED CONTENT}

\section{s) Supporting Information}

The Supporting Information is available free of charge at https://pubs.acs.org/doi/10.1021/acs.joc.1c00235.

LC-MS total ion current chromatograms and ${ }^{1} \mathrm{H}$ and ${ }^{13} \mathrm{C}$ NMR spectra of compounds (PDF)

FAIR data, including the primary NMR FID files, for compounds 6-8, 11-18, 20, 22-35, 37, and 38 (ZIP)

\section{AUTHOR INFORMATION}

\section{Corresponding Author}

Mikael Elofsson - Department of Chemistry, Umea University, Umeå SE90187, Sweden; ㅇo이.org/00000002-3219-4669; Email: mikael.elofsson@umu.se

\section{Authors}

Emil Johansson - Department of Chemistry, Umeå University, Umea SE90187, Sweden

Rémi Caraballo - Department of Chemistry, Umea University, Umeå SE90187, Sweden; 다이.org/0000-0001-59128429

Complete contact information is available at:

https://pubs.acs.org/10.1021/acs.joc.1c00235

\section{Author Contributions}

All authors designed the study. E.J. and R.C. performed the experimental work, and all authors analyzed and interpreted data. E.J. wrote the manuscript with contributions of all authors. All authors have given approval to the final version of the manuscript. E.J. and R.C. contributed equally.

\section{Funding}

The project was funded by a Knut and Alice Wallenberg Foundation grant (KAW; grant reference no. 2013.0019).

\section{Notes}

The authors declare no competing financial interest.

\section{REFERENCES}

(1) Varki, A. Sialic acids in human health and disease. Trends Mol. Med. 2008, 14, 351-360.

(2) Suzuki, Y. Sialobiology of influenza: Molecular mechanism of host range variation of influenza viruses. Biol. Pharm. Bull. 2005, 28, 399-408.

(3) Wu, X.; Wu, X.; Sun, Q.; Zhang, C.; Yang, S.; Li, L.; Jia, Z. Progress of small molecular inhibitors in the development of antiinfluenza virus agents. Theranostics 2017, 7, 826-845.

(4) von Itzstein, M.; Jin, B.; Wu, W.-Y.; Chandler, M. A convenient method for the introduction of nitrogen and sulfur at C-4 on a sialic acid analogueue. Carbohydr. Res. 1993, 244, 181-185.

(5) von Itzstein, M.; Wu, W.-Y.; Kok, G. B.; Pegg, M. S.; Dyason, J. C.; Jin, B.; Van Phan, T.; Smythe, M. L.; White, H. F.; Oliver, S. W.; Colman, P. M.; Varghese, J. N.; Ryan, D. M.; Woods, J. M.; Bethell, R. C.; Hotham, V. J.; Cameron, J. M.; Penn, C. R. Rational design of potent sialidase-based inhibitors of influenza virus replication. Nature 1993, 363, 418-423.

(6) Kok, G. B.; Groves, D.; von Itzstein, M. A facile synthesis of Neu5Ac2en derivatives from the glycosides of N-acetylneuraminic acid. J. Chem. Soc., Perkin Trans. 1 1999, 2109-2116.

(7) Schreiner, E.; Zbiral, E.; Kleineidam, R. G.; Schauer, R. Structural variations on $\mathrm{N}$-acetylneuraminic acid, 20. Synthesis of some 2,3-didehydro-2-deoxysialic acids structurally varied at C-4 and their behavior towards sialidase from Vibrio cholerae. Liebigs Ann. 1991, 1991, 129-134.

(8) Starkey, I. D.; Mahmoudian, M.; Noble, D.; Smith, P. W.; Cherry, P. C.; Howes, P. D.; Sollis, S. L. Synthesis and influenza virus 
sialidase inhibitory activity of the 5-desacetamido analogueue of 2,3didehydro-2,4-dideoxy-4-guanidinyl- $\mathrm{N}$-acetylneuraminic acid (GG167). Tetrahedron Lett. 1995, 36, 299-302.

(9) Kok, G. B.; von Itzstein, M. A regio- and stereo-selective introduction of azide at $\mathrm{C}-4$ of 2,3-unsaturated $\mathrm{N}$-acetylneuraminic acids. Carbohydr. Res. 1997, 302, 237-240.

(10) Liu, K.-G.; Yan, S.; Wu, Y.-L.; Yao, Z.-J. Synthesis of 4-Azido-4deoxy-Neu5,7,8,9Ac42en1Me. A key intermediate for the synthesis of GG167 from d-glucono- $\delta$-lactone. Org. Lett. 2004, 6, 2269-2272.

(11) Hemeon, I.; Bennet, A. J. An unexpected elimination product leads to 4-alkyl-4-deoxy-4-epi-sialic acid derivatives. Can. J. Chem. 2008, 86, 238-247.

(12) Ye, D.; Li, J.; Zhang, J.; Liu, H.; Jiang, H. Simultaneous stereoselective 4-amination with cyclic secondary amines and 2-Odeacetylation of peracetylated sialic acid derivatives. Tetrahedron Lett. 2007, 48, 4023-4027.

(13) Kok, G. B.; van Phan, T.; von Itzstein, M. Bromohydroxylation of glycals - An investigation into the reaction of some 4-N-acylated derivatives of methyl 5-acetamido-7,8,9-tri-O-acetyl-2,6-anhydro3,4,5-trideoxy-D-glycero-D-galacto-non-2-enonate and its 4-epimer. J. Carbohydr. Chem. 2001, 20, 359-374.

(14) Shidmoossavee, F. S.; Watson, J. N.; Bennet, A. J. Chemical insight into the emergence of influenza virus strains that are resistant to Relenza. J. Am. Chem. Soc. 2013, 135, 13254-13257.

(15) Buda, S.; Crich, D. Oxidative deamination of N-acetyl neuraminic acid: Substituent effects and mechanism. J. Am. Chem. Soc. 2016, 138, 1084-1092.

(16) Hartmann, M.; Christian, R.; Zbiral, E. Structural variations of $\mathrm{N}$-acetylneuraminic acid, part 19: Synthesis of both epimeric pairs of the 4-C-methyl- and 4-deoxy-4-C-methyl- as well as of the $\beta$ methylketoside of 4-deoxy-4-C-methylene- $\mathrm{N}$-acetylneuraminic acid. Monatsh. Chem. 1991, 122, 111-125.

(17) Hasegawa, A.; Adachi, K.; Yoshida, M.; Kiso, M. Synthetic studies on sialoglycoconjugates 31: Synthesis of ganglioside GM3 analogues containing the chemically modified sialic acids. $J$. Carbohydr. Chem. 1992, 11, 95-116.

(18) Liav, A. Alkylation of $\mathrm{N}$-acetylneuraminic acid at C-4. Carbohydr. Res. 1995, 271, 241-245.

(19) Ikeda, K.; Sano, K.; Ito, M.; Saito, M.; Hidari, K.; Suzuki, T.; Suzuki, Y.; Tanaka, K. Synthesis of 2-deoxy-2,3-didehydro-Nacetylneuraminic acid analogueues modified at the C-4 and C-9 positions and their behaviour towards sialidase from influenza virus and pig liver membrane. Carbohydr. Res. 2001, 330, 31-41.

(20) Ikeda, K.; Kimura, F.; Sano, K.; Suzuki, Y.; Achiwa, K. Chemoenzymatic synthesis of an $\mathrm{N}$-acetylneuraminic acid analogueue having a carbamoylmethyl group at C-4 as an inhibitor of sialidase from influenza virus. Carbohydr. Res. 1998, 312, 183-189.

(21) Baumberger, F.; Vasella, A. Deoxy-nitrosugars. 16th Communication. Synthesis of N-acetyl-4-deoxyneuraminic acid. Helv. Chim. Acta 1986, 69, 1535-1541.

(22) Li, Y.-F.; Maliakel, B. P.; Zbiral, E. Formation of a lactam derivative on direct benzylation of $\mathrm{N}$-acetylneuraminic acid $\alpha$-methyl ketoside 1. Synlett 1992, 1992, 561-562.

(23) Yuan, X.; Ress, D. K.; Linhardt, R. J. Synthesis of nor-C-linked neuraminic acid disaccharide: $\mathrm{A}$ versatile precursor of $\mathrm{C}$-analogues of oligosialic acids and gangliosides. J. Org. Chem. 2007, 72, 3085-3088.

(24) Wallimann, K.; Vasella, A. C-Glykoside der N-Acetylneuraminsäure. Synthese und Untersuchung ihrer Wirkung auf die Vibrio cholerae Sialidase. Helv. Chim. Acta 1991, 74, 1520-1532.

(25) Sugiyama, N.; Sugai, K. E. I.; Yamada, N.; Goto, M.; Ban, C.; Furuhata, K.; Takayanagi, H.; Ogura, H. Studies on sialic acids. IX.: Formation of a 1, 7-lactone derivative by direct acetylation of $\mathrm{N}$ acetylneuraminic acid. Chem. Pharm. Bull. 1988, 36, 1147-1152.

(26) Wallimann, K.; Vasella, A. Phosphonic-acid analogueues of the N-acetyl-2-deoxyneiiraniinic acids: Synthesis and inhibition of Vibrio cholerae sialidase. Helv. Chim. Acta 1990, 73, 1359-1372.

(27) Sharma, M.; Petrie, C. R.; Korytnyk, W. General methods for modification of sialic acid at C-9. Synthesis of N-acetyl-9-deoxy-9fluoroneuraminic acid. Carbohydr. Res. 1988, 175, 25-34.
(28) Miyazaki, T.; Sato, H.; Sakakibara, T.; Kajihara, Y. An approach to the precise chemoenzymatic synthesis of 13C-labeled sialyloligosaccharide on an intact glycoprotein: A novel one-pot [3-13C]labeling method for sialic acid analogueues by control of the reversible aldolase reaction, enzymatic synthesis of [3-13C]-NeuAc- $\alpha-(2 \rightarrow 3)$ $[\mathrm{U}-13 \mathrm{C}]$-Gal- $\beta$-( $1 \rightarrow 4)$-GlcNAc- $\beta$-sequence onto glycoprotein, and its conformational analysis by developed NMR techniques. J. Am. Chem. Soc. 2000, 122, 5678-5694.

(29) Mesch, S.; Lemme, K.; Koliwer-Brandl, H.; Strasser, D. S.; Schwardt, O.; Kelm, S.; Ernst, B. Kinetic and thermodynamic properties of MAG antagonists. Carbohydr. Res. 2010, 345, 13481359.

(30) Hanashima, S.; Ishikawa, D.; Akai, S.; Sato, K.-i. Synthesis of the starfish ganglioside LLG-3 tetrasaccharide. Carbohydr. Res. 2009, $344,747-752$.

(31) Nilsson, E. C.; Storm, R. J.; Bauer, J.; Johansson, S. M. C.; Lookene, A.; Ångström, J.; Hedenström, M.; Eriksson, T. L.; Frängsmyr, L.; Rinaldi, S.; Willison, H. J.; Domellöf, F. P.; Stehle, T.; Arnberg, N. The GD1a glycan is a cellular receptor for adenoviruses causing epidemic keratoconjunctivitis. Nat. Med. 2011, 17, 105-109.

(32) Caraballo, R.; Saleeb, M.; Bauer, J.; Liaci, A. M.; Chandra, N.; Storm, R. J.; Frängsmyr, L.; Qian, W.; Stehle, T.; Arnberg, N.; Elofsson, M. Triazole linker-based trivalent sialic acid inhibitors of adenovirus type 37 infection of human corneal epithelial cells. Org. Biomol. Chem. 2015, 13, 9194-9205.

(33) Spjut, S.; Qian, W.; Bauer, J.; Storm, R.; Frängsmyr, L.; Stehle, T.; Arnberg, N.; Elofsson, M. A potent trivalent sialic acid inhibitor of adenovirus type 37 infection of human corneal cells. Angew. Chem., Int. Ed. 2011, 50, 6519-6521.

(34) Zocher, G.; Mistry, N.; Frank, M.; Hähnlein-Schick, I.; Ekström, J.-O.; Arnberg, N.; Stehle, T. A sialic acid binding site in a human picornavirus. PLoS Pathog. 2014, 10, e1004401.

(35) Johansson, E.; Caraballo, R.; Mistry, N.; Zocher, G.; Qian, W.; Andersson, C. D.; Hurdiss, D. L.; Chandra, N.; Thompson, R.; Frängsmyr, L.; Stehle, T.; Arnberg, N.; Elofsson, M. Pentavalent sialic acid conjugates llock coxsackievirus A24 variant and human adenovirus type 37 - viruses that cause highly contagious eye infections. ACS Chem. Biol. 2020, 15, 2683-2691.

(36) Tornøe, C. W.; Christensen, C.; Meldal, M. Peptidotriazoles on solid phase: $[1,2,3]$-triazoles by regiospecific copper(I)-catalyzed 1,3dipolar cycloadditions of terminal alkynes to azides. J. Org. Chem. 2002, 67, 3057-3064.

(37) Rostovtsev, V. V.; Green, L. G.; Fokin, V. V.; Sharpless, K. B. A stepwise Huisgen cycloaddition process: Copper(I)-catalyzed regioselective "ligation" of azides and terminal alkynes. Angew. Chem., Int. Ed. 2002, 41, 2596-2599.

(38) Ogura, H.; Furuhata, K.; Itoh, M.; Shitori, Y. Syntheses of 2-Oglycosyl derivatives of $\mathrm{N}$-acetyl-d-neuraminic acid. Carbohydr. Res. 1986, 158, 37-51.

(39) Anazawa, K.; Furuhata, K.; Ogura, H. Synthesis of 7-O-AcetylN-acetylneuraminic Acid Derivative. Chem. Pharm. Bull. 1988, 36, 4976-4979.

(40) Kim, J.-H.; Huang, F.; Ly, M.; Linhardt, R. J. Stereoselective synthesis of a C-linked neuraminic acid disaccharide: Potential building block for the synthesis of C-analogueues of polysialic acids. J. Org. Chem. 2008, 73, 9497-9500.

(41) Liu, D.-M.; Wang, H.-L.; Lei, J.-C.; Zhou, X.-Y.; Yang, J.-S. A highly $\alpha$-stereoselective sialylation method using 4-O-4-nitropicoloyl thiosialoside donor. Eur. J. Org. Chem. 2020, 2020, 575-585.

(42) Magnera, T. F.; Caldwell, G.; Sunner, J.; Ikuta, S.; Kebarle, P. Solvation of the halide anions in dimethyl sulfoxide. Factors involved in enhanced reactivity of negative ions in dipolar aprotic solvents. J. Am. Chem. Soc. 1984, 106, 6140-6146.

(43) Yang, L.; Zhang, J.; Xie, J.; Ma, X.; Zhang, L.; Zhao, C.; Hase, W. L. Competing E2 and SN2 mechanisms for the F- + CH3CH2I Reaction. J. Phys. Chem. A 2017, 121, 1078-1085. 
(44) De Meo, C.; Wallace, C. E.; Geringer, S. A. Synthesis and elimination of C-3-labeled thiosialosides. Org. Lett. 2014, 16, 26762679.

(45) Suzuki, T.; Ikeda, K.; Koyama, N.; Hosokawa, C.; Kogure, T.; Takahashi, T.; Jwa Hidari, K. I. P.; Miyamoto, D.; Tanaka, K.; Suzuki, Y. Inhibition of human parainfluenza virus type 1 sialidase by analogues of 2-deoxy-2,3-didehydro-N-acetylneuraminic acid. Glycoconjugate J. 2001, 18, 331-337.

(46) Ikeda, K.; Sato, K.; Kitani, S.; Suzuki, T.; Maki, N.; Suzuki, Y.; Sato, M. 2-Deoxy-2,3-didehydro-N-acetylneuraminic acid analogueues structurally modified at the C-4 position: Synthesis and biological evaluation as inhibitors of human parainfluenza virus type 1. Bioorg. Med. Chem. 2006, 14, 7893-7897.

(47) Tindal, D. J.; Dyason, J. C.; Thomson, R. J.; Suzuki, T.; Ueyama, H.; Kuwahara, Y.; Maki, N.; Suzuki, Y.; Itzstein, M. v. Synthesis and evaluation of 4-O-alkylated 2-deoxy-2,3-didehydro-Nacetylneuraminic acid derivatives as inhibitors of human parainfluenza virus type-3 sialidase activity. Bioorg. Med. Chem. Lett. 2007, 17, $1655-1658$.

(48) Sato, K.; Ikeda, K.; Suzuki, T.; Aoyama, S.; Maki, N.; Suzuki, Y.; Sato, M. Synthesis of 4-O-[3-(aryl)prop-2-ynyl]-Neu5Ac2en and its 4-epi-analogues modified at C-4 by Sonogashira coupling reaction. Tetrahedron 2007, 63, 7571-7581.

(49) Ikeda, K.; Sato, K.; Nishino, R.; Aoyama, S.; Suzuki, T.; Sato, M. 2-Deoxy-2,3-didehydro-N-acetylneuraminic acid analogues structurally modified by thiocarbamoylalkyl groups at the C-4 position: Synthesis and biological evaluation as inhibitors of human parainfluenza virus type 1. Bioorg. Med. Chem. 2008, 16, 6783-6788.

(50) Ando, H.; Shimizu, H.; Katano, Y.; Koike, Y.; Koizumi, S.;

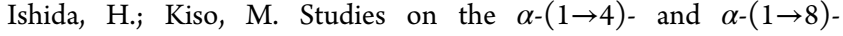
fucosylation of sialic acid for the total assembly of the glycan portions of complex HPG-series gangliosides. Carbohydr. Res. 2006, 341 (10), $1522-1532$.

(51) Johansson, E.; Caraballo, R.; Elofsson, M. Synthesis of 4-OAlkylated N-Acetylneuraminic Acid Derivatives. 2020. ChemRxiv Preprint. https://chemrxiv.org/articles/preprint/Synthesis_of_4-OAlkylated_N-Acetylneuraminic_Acid_Derivatives/13372289/1. 